\title{
Xuesaitong injection (lyophilized) combined with aspirin and clopidogrel protect against focal cerebral ischemic/reperfusion injury in rats by suppressing oxidative stress and inflammation and regulating the NOX2/IL-6/STAT3 pathway
}

\author{
Ting Zhu ${ }^{1}$, Xiang-Bao Meng ${ }^{1}$, Dong-Xia Dong ${ }^{1}$, Li-Yu Zhao ${ }^{1,2}$, Mu-Wen Qu ${ }^{3}$, Gui-Bo Sun ${ }^{1}$, Xiao-Bo Sun ${ }^{1}$ \\ ${ }^{1}$ Beijing Key Laboratory of Innovative Drug Discovery of Traditional Chinese Medicine (Natural Medicine) and Translational Medicine, Institute of \\ Medicinal Plant Development, Peking Union Medical College and Chinese Academy of Medical Sciences; Key Laboratory of Bioactive Substances \\ and Resources Utilization of Chinese Herbal Medicine, Ministry of Education, Institute of Medicinal Plant Development, Chinese Academy of \\ Medical Sciences \& Peking Union Medical College; Key Laboratory of New Drug Discovery Based on Classic Chinese Medicine Prescription, \\ Chinese Academy of Medical Sciences, Beijing, China; ${ }^{2}$ Harbin University of Commerce, Heilongiiang, China; ${ }^{3}$ Guang'anmen Hospital of Chinese \\ Academy of Chinese Medical Sciences, Beijing, China \\ Contributions: (I) Conception and design: T Zhu, XB Meng; (II) Administrative support: XB Sun; (III) Provision of study materials or patients: T \\ Zhu, XB Meng; (IV) Collection and assembly of data: XB Meng, T Zhu, GB Sun; (V) Data analysis and interpretation: All authors; (VI) Manuscript \\ writing: All authors; (VII) Final approval of manuscript: All authors. \\ Correspondence to: Xiao-Bo Sun. Institute of Medicinal Plant Development, Peking Union Medical College and Chinese Academy of Medical \\ Sciences, Beijing 100193, China. Email: sun-xiaobo@163.com; Gui-Bo Sun. Institute of Medicinal Plant Development, Peking Union Medical \\ College and Chinese Academy of Medical Sciences, Beijing 100193, China. Email: sunguibo@126.com; Mu-Wen Qu. Guang'anmen Hospital of \\ Chinese Academy of Chinese Medical Sciences, Beijing 100053, China. Email: 776992400@qq.com.
}

Background Combination of aspirin (ASA) and clopidogrel (CLP) [dual antiplatelet therapy (DAPT)] has been limited in reducing early recurrent stroke events. Xuesaitong injection (lyophilized) (XST) made of total saponins from $P$. notoginseng, which significantly improves cerebral circulation and has been widely used in clinical applications for decades to treat and prevent ischemic stroke. Here, we confirmed the protective effect and mechanism of XST combined with DAPT (XST+ASA+CLP) on cerebral ischemia/reperfusion injury, exploring their better pharmacological action for clinical patients.

Methods: Sprague-Dawley rats (SD rats) ( $\mathrm{n}=9$ in each group) were randomly assigned to three groups and pretreated with XST, ASA+CLP, or XST+ASA+CLP for 7 days. Then rats were subjected to $2 \mathrm{~h}$ of middle cerebral artery occlusion (MCAO) followed by reperfusion for $24 \mathrm{~h}$. Therapeutic effect of XST+ASA+CLP was measured by infarct volume, neurological behavior and regional cerebral blood flow (rCBF). Inhibition of neuronal apoptosis and glial cells was determined by immunofluorescent staining. We studied the protein levels of neurotrophic factors, neuroplasticity-related factors, oxidative stress indicators and inflammatory factors by ELISA assay.

Results: XST+ASA+CLP group showed significant reduction in infarct volumes and neurological deficit scores. XST+ASA+CLP group also had higher levels in rCBF and synaptic growth, and showed remarkable inhibition of microglia and astrocytes activation and the neuronal apoptosis. In addition, XST+ASA+CLP group had lower levels of NADPH, protein carbonyl, 4-hydroxynonenal (4-HNE), 8-hydroxydeoxyguanosine (8-OHdG) and several inflammatory cytokines. Moreover, XST+ASA+CLP group also had lower levels of NOX2, inducible nitric oxide synthase (iNOS), interleukin (IL)-6, and p-STAT3/STAT3.

Conclusions: These results demonstrate that a combination of XST, ASA, and CLP effectively protected rats against middle cerebral artery occlusion/reperfusion (MCAO/R) injury by suppressing the NOX2/IL-6/ STAT3 pathway. These novel findings provide theoretical basis and experimental evidence for the rationality of clinical combined use of drugs in the treatment of ischemic stroke.

Keywords: Xuesaitong injection; aspirin (ASA); clopidogrel (CLP); oxidative stress; inflammation 
Submitted May 01, 2020. Accepted for publication Oct 15, 2020.

doi: 10.21037/apm-20-1681

View this article at: http://dx.doi.org/10.21037/apm-20-1681

\section{Introduction}

Stroke is a cerebrovascular disease that causes irreversible neurological damage and remains one of the leading causes of death and disability in adults worldwide (1). Various pharmacological agents, which target neuronal death blockade or neuronal survival and regeneration enhancement after stroke, have been the bases of neuroprotection research (2). Therapeutics targeting neuronal death blockade are currently identified as the basic way to overcome the limitations of stroke treatments. However, effective antagonists that treat stroke are very limited in clinical and basic research.

Multiple mechanisms, including excitotoxicity, inflammation, oxidative stress, apoptosis, calcium influx, and mitochondria injury, are key contributors to brain damage after stroke (2). Inflammation caused by stroke may contribute to neural dysfunction, cell death, and poor clinical outcomes (3). As a resident immune cell of the central nervous system (CNS), phagocytic microglia maintain immune homeostasis primarily through regulated pro- and anti-inflammatory signaling pathways (4). Interestingly, ischemic stroke increases the production of early pro-inflammatory cytokines, leading to deep immunosuppression and bilateral peripheral inflammatory responses (5). Furthermore, ischemic tissue reperfusion aggravates cerebral injury caused by oxidative stress, which generated excessive reactive oxygen species (ROS) (6). The role of ROS sources should be clarified because of their involvement in oxidative stress. Nicotinamide adenosine dinucleotide phosphate (NADPH) oxidases are the major sources of ROS in the pathological process of cerebral ischemia/reperfusion (I/R) injury $(7,8)$. NADPH oxidase $(\mathrm{NOX})$ is a multiunit enzyme originally discovered in neutrophils and has recently been proved to be a major producer of ROS and oxidative stress (9). Our in vitro study showed that notoginsenoside R1 (NG-R1) exhibited neuroprotection against cerebral I/R injury by inhibiting NOX activity via endoplasmic reticulum (ER)-dependent activation of the Akt/Nrf2 pathway (10). At present, five NOX enzyme isoforms (NOX 1-5) have been identified and localized. NOX2, now known as gp91phox, and NOX4, are primarily localized in the cerebral cortex and hippocampus
CA1 $(11,12)$. The infarct sizes significantly decreased in NOX2 inhibitor-treated animals and NOX2 knockout animals $(13,14)$, therefore NOX2 is extremely important in oxidative stress-induced neuron damage following stroke. However, whether NOX inhibition reduces neuroinflammation and improves neurological function in ischemic stroke is largely undefined.

Aspirin (ASA) is a prophylactic antiplatelet drug for people with cardiovascular and cerebrovascular diseases, such as heart attack and stroke, in high-risk populations with thrombosis. The antithrombotic action of ASA works by preventing the biosynthesis of thromboxane-A2 (TXA2) by inactivating its reversible COX-1, which inhibits platelet aggregation (15). Clopidogrel (CLP) is an oral antiplatelet agent used to inhibit thrombosis in coronary artery disease, cerebrovascular disease, and peripheral vascular disease. The anti-aggregation activity of CLP is attributed to short-acting metabolites that are produced by the cytochrome P450-dependent pathway in the liver, which prevents the binding of adenosine diphosphate (ADP) to its platelet receptor (16). Long-term use of antiplatelet drugs in patients with noncardiac embolic stroke or transient ischemic attack, are crucial for extended secondary prevention of stroke and other cardiovascular events; such drugs include ASA monotherapy, CLP monotherapy and combination of ASA and extended-release dipyridamole treatment (17). Moreover, the American Heart Association and the American Stroke Association have issued updated clinical practice guidelines on dual antiplatelet therapy (DAPT). Based on these guidelines, DAPT with ASA and CLP provided additional benefits in reducing the occurrence of numerous vascular events and inhibiting intense platelet aggregation in patients with early recurrent stroke and coronary artery disease (18-20). However, due to the ceiling effect and unresolved bleeding risk during the treatment process, DAPT therapy is in trouble.

At present, Chinese patent medicines for treating acute ischemic stroke are mainly drugs for promoting blood circulation and removing blood stasis. Panax notoginseng, also known as Sanqi, is an ancient Chinese medicinal plant that has great clinical value to regulate cardiovascular (21) and neurological diseases (22) in China. Xuesaitong 

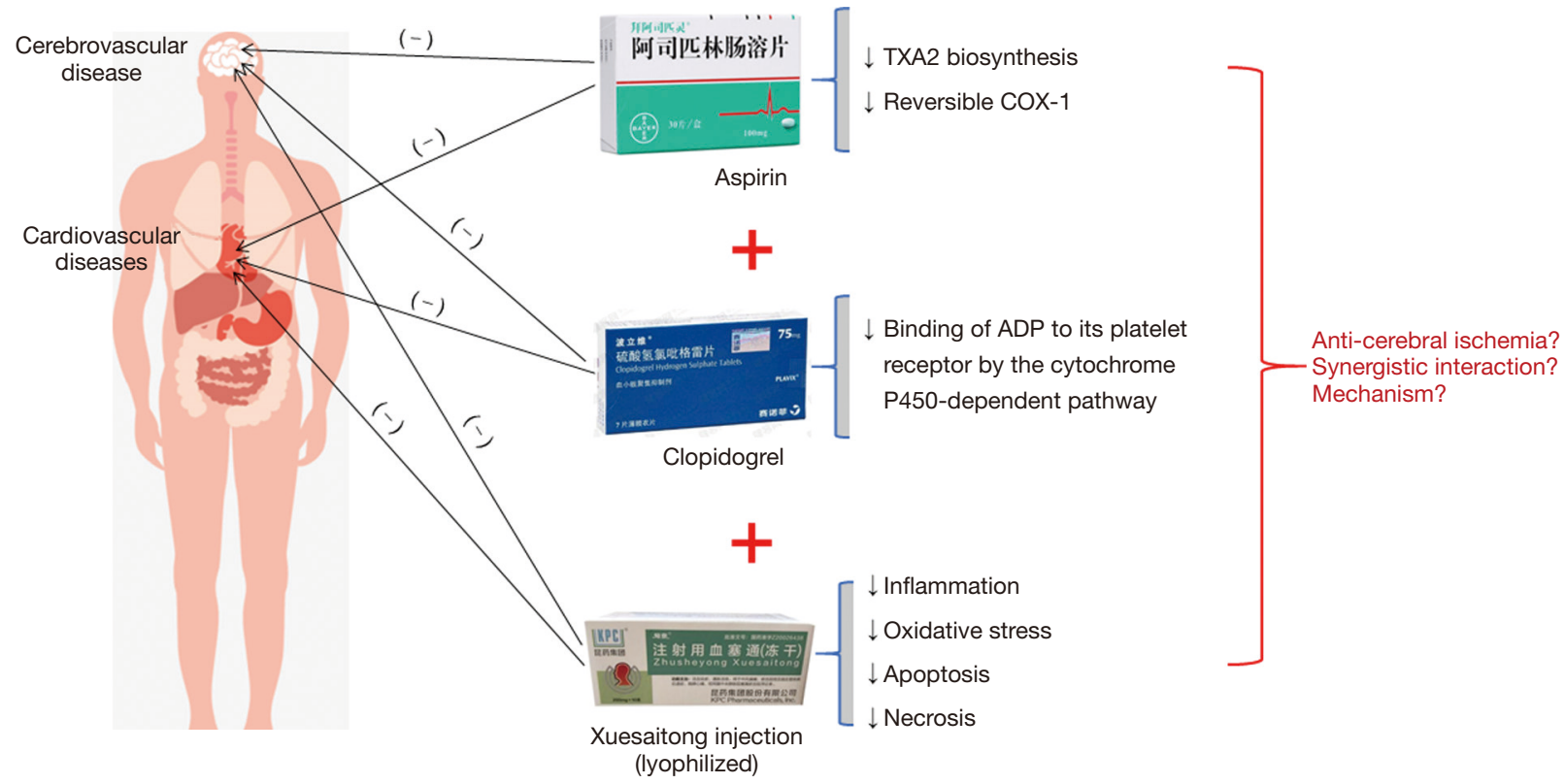

Figure 1 Summary of the pharmacological effects and mechanisms of ASA, CLP and XST. (-) ( $\downarrow)$, inactivation or inhibition. XST, Xuesaitong injection (lyophilized); ASA, aspirin; CLP, clopidogrel.

injection (lyophilized) (XST) made of total saponins from $P$. notoginseng, which significantly improves cerebral circulation and has been widely used in clinical applications for decades to treat and prevent ischemic stroke. In the past few decades, more than ten million patients have been cured by injection of XST. Our previous study showed that Xuesaitong capsules combined with ASA attenuates brain dysfunction in rats with ischemic stroke by inhibiting oxidative stress and inflammatory response (23). Other studies have also demonstrated that panax notoginsenoside (PNS) reduces the neurological deficit and cerebral infarct volume and protects the nervous system from I/R injury by inhibiting inflammation, oxidative stress, cell apoptosis, and necrosis $(24,25)$. However, whether the combination of DAPT and XST are superior to XST monotherapy for ischemic stroke remains largely unknown. In the present study, we first investigated the effect of combination of XST+ASA+CLP (ASA $10 \mathrm{mg} / \mathrm{kg}$, CLP $7.5 \mathrm{mg} / \mathrm{kg}$ plus XST $40 \mathrm{mg} / \mathrm{kg}$ ) against inflammation and oxidative stress through the downstream pathway, NOX2/IL-6/STAT3, after ischemic stroke. These benefits provide experimental evidence for clinical precise positioning and rational drug use (Figure 1). We present the following article in accordance with the ARRIVE reporting checklist (available at http://dx.doi.org/10.21037/apm-20-1681).

\section{Methods}

\section{Animals}

Male Sprague-Dawley (SD) rats weighing $300 \pm 10 \mathrm{~g}$ were purchased from Beijing Vital River Laboratories and used in this study. Five rats were housed per cage on a 12-h artificial light and given free access to food and sterilized drinking water in an animal facility with controlled temperature $\left(20-25^{\circ} \mathrm{C}\right)$ and humidity $(30-50 \%)$. All animal care and experimental procedures were reported in accordance with the Institutional Animal Care and Use Committee of the Chinese Academy of Medical Sciences \& Peking Union Medical College and complied with NIH Guidelines for the Care and Use of Laboratory Animals (approval number: SYXK 2017-0020). All efforts were followed to reduce the number of animals used and ensure minimal suffering.

\section{MCAO surgery and regional cerebral blood flow (rCBF) measurement}

$\mathrm{SD}$ rats were anaesthetized with ketamine $(80 \mathrm{mg} / \mathrm{kg})$ and xylazine $(10 \mathrm{mg} / \mathrm{kg})$ via intraperitoneal injections. Middle cerebral artery occlusion/reperfusion $(\mathrm{MCAO} / \mathrm{R})$ was proceeded by experimenters who were unaware of the grouping scheme, following a previously described method (10). 
Rats in the sham group were operated using the same method but with no occlusion in middle cerebral artery. The body temperature of rats was maintained at $37 \pm 0.5{ }^{\circ} \mathrm{C}$ during the MCAO procedure with a heating pad (Sunbeam, USA). The incision was sewed after ischemia and cerebral blood flow in the ischemic core region and peripheral region after reperfusion $24 \mathrm{~h}$ was assessed by the laser Doppler flowmeter (moor FLPI-2, UK).

\section{Drug treatment}

XST was provided by Kunmingshenghuo Pharmaceutical Co., Ltd.; ASA was obtained from Bayer Medical and Health Co., Ltd.; and CLP was purchased from Sanofi Pharmaceutical Co., Ltd. (Hangzhou, China). Prior to administration, the drugs were freshly prepared in normal saline. Male SD rats were randomly assigned to five groups, which were pre-treated for 7 days before $\mathrm{MCAO} / \mathrm{R}$. The sham and MCAO/R groups were given normal saline solution injection intravenously (i.v.) daily. The XST group was treated with XST at a dose of $40 \mathrm{mg} / \mathrm{kg}$ i.v. through the tail once a day. The ASA and CLP co-treatment groups (ASA+CLP) were treated with ASA at a dose of $10 \mathrm{mg} / \mathrm{kg}$ intragastrically (i.g.) and followed by CLP at a dose of $7.5 \mathrm{mg} / \mathrm{kg}$ i.g. every day. The XST, ASA, and CLP co-treatment groups (XST+ASA+CLP) were treated with $40 \mathrm{mg} / \mathrm{kg}$ XST i.v., $10 \mathrm{mg} / \mathrm{kg}$ ASA i.g., and $7.5 \mathrm{mg} / \mathrm{kg}$ CLP i.g. After 7 days, serum, hippocampus, and cortex tissues were collected for mechanism research underlying ischemic stroke in $\mathrm{XST}+\mathrm{ASA}+\mathrm{CLP}$ intervention in rats with $\mathrm{MCAO} / \mathrm{R}$.

\section{Neurological score}

Neurological performances of all animals were evaluated by two blinded investigators using Zea Longa Scores following a previously described method (10). The neurological function was scored according to a series of scales from 0 to 4. The highest score represents the most severe neurological deficits.

\section{Triphenyltetrazolium chloride (TTC) staining}

TTC staining was conducted $24 \mathrm{~h}$ post-stroke based on the methods described previously ( $\mathrm{n}=3$ for each group) $(10,26)$. Cerebral infarct area was quantified by an image analysis system (Image-Pro Plus 5.0). The infarct volume can be obtained by multiplying the total infarct area by the thickness of the brain sections. Calculating the corrected infarct volume is contribute to compensate for the error caused by brain edema (10).

\section{Western blot analysis}

Western blot method was conducted as previously reported (10). The primary antibodies used in the experiment were as follows: NOX2 antibody (Abcam, USA), inducible nitric oxide synthase (iNOS) antibody (Cell Signaling Technology, USA), anti-IL-6 (Abcam, USA), anti-STAT3 (Abcam, USA), and anti-phospho-STAT3 (Abcam, USA). Blot densities were calculated by ImageJ software.

\section{Enzyme-linked immunosorbent assay (ELISA)}

ELISA kits (Boster Biotechnology, China) were used according to the manufacturer's instructions to quantify the expression of the oxidative stress markers NADPH, protein carbonyl, 4-hydroxynonenal (4HNE), 8-hydroxydeoxyguanosine (8-OHdG), and proinflammatory cytokines TNF- $\alpha$, IL- $1 \beta$, IL-6, ICAM1, CD11a, MCP-1, IL-10, and IL-4. In brief, serum and homogenates of the right cortex and hippocampus were prepared for analysis. Oxidative stress markers and inflammatory cytokine levels were expressed as a percentage of the control.

\section{Immunofluorescent staining}

Immunofluorescence staining was implemented as previously reported (27). Primary antibodies, namely rabbit monoclonal NeuN antibody, rabbit monoclonal GFAP antibody, and mouse monoclonal Iba-1 antibody, were purchased from Abcam, USA for the experiment. Images were obtained through at least three random visual fields from three separate sections of each sample by using fluorescence microscope (Carl Zeiss, Germany) at magnification of $200 \times$. Mean values of Ib $\alpha-1$ and GFAP were calculated for statistical analysis.

\section{TUNEL staining}

Neuronal apoptosis in brain tissue was evaluated by TUNEL staining with the neural marker, NeuN (Abcam, USA) using a TUNEL kit (Beyotime Institute of Biotechnology, China) as previously reported (26). The images of TUNEL positive neuronal cells were captured using a fluorescence microscope (Carl Zeiss, Germany). 
A

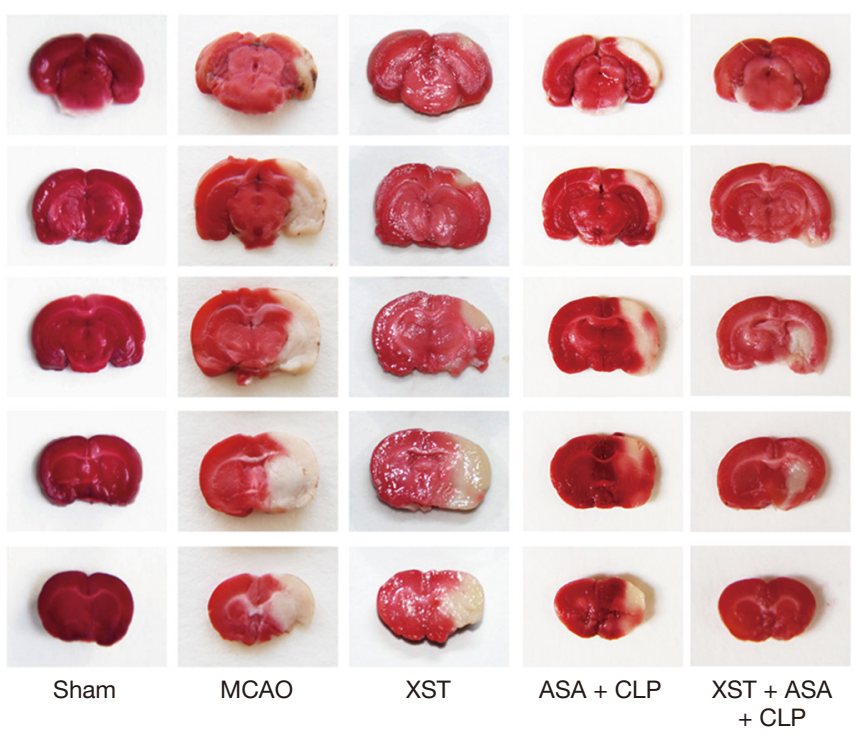

B

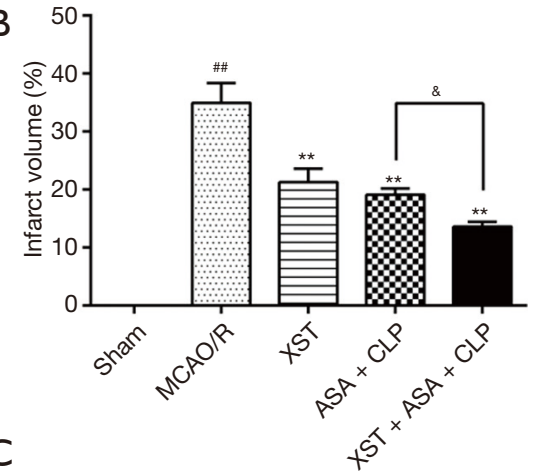

C

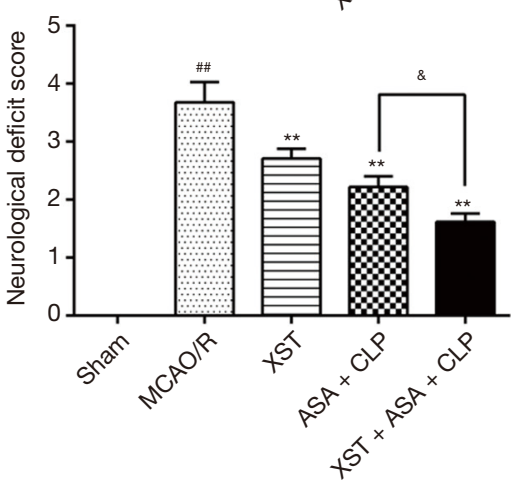

Figure $2 \mathrm{XST}+\mathrm{ASA}+\mathrm{CLP}$ attenuates MCAO/R-induced infarction volumes and neurologic deficits. (A) Images of TTC staining at $24 \mathrm{~h}$ after reperfusion; (B) quantitative analysis of cerebral infarct volumes; (C) neurological score in rats with MCAO/R at $24 \mathrm{~h}$ after reperfusion. **, $\mathrm{P}<0.01$, compared with the MCAO/R group; ${ }^{\# \#, ~} \mathrm{P}<0.01$, compared with the sham group; ${ }^{\circ}, \mathrm{P}<0.05$, compared with the ASA+CLP group. XST, Xuesaitong injection (lyophilized); ASA, aspirin; CLP, clopidogrel; MCAO/R, middle cerebral artery occlusion/reperfusion; TTC: 2,3,5-triphenyltetrazoliumchloride.

\section{Statistical analysis}

All statistical analyses were performed with GraphPad Prism 6.0 (SPAA, Inc., Chicago, Illinois, USA). Data were expressed as means \pm standard deviation (SD) and analyzed by one-way ANOVA followed by Tukey's test. Differences were compared between two groups using Student's $t$-test. $\mathrm{P}<0.05$ was considered statistically significant.

\section{Results}

\section{$X S T+A S A+C L P$ attenuates MCAO/R-induced infarction volumes and neurologic deficits}

At $24 \mathrm{~h}$ after reperfusion, TTC staining and neurologic deficit score were used to measured infarction and neurological function. As shown in Figure $2 A, B$, the rats that received MCAO treatment revealed a well-demarcated infarct in ischemic core and penumbra $(\mathrm{P}<0.01)$. Compared with the MCAO/R group, the XST and ASA+CLP groups exhibited significant decrease in infarction $(\mathrm{P}<0.01)$.
Furthermore, the XST+ASA+CLP co-treated group showed significant changes in infarction compared with the ASA+CLP co-treated group $(\mathrm{P}<0.05)$. Meanwhile, a visible amelioration in neurologic deficit was observed in $\mathrm{XST}+\mathrm{ASA}+\mathrm{CLP}$ co-treated rats (Figure $2 C, \mathrm{P}<0.05$ ).

\section{$X S T+A S A+C L P$ increases $r C B F$}

As shown in Figure $3 A, B$, the rCBF in different groups was evaluated using a laser Doppler flowmeter system. The results revealed that the administration of XST, ASA+CLP, and $\mathrm{XST}+\mathrm{ASA}+\mathrm{CLP}$ in treated groups resulted in a significant increase in $\mathrm{rCBF}$ at $24 \mathrm{~h}$ after reperfusion versus the $\mathrm{MCAO} / \mathrm{R}$ group $(\mathrm{P}<0.01)$. The $\mathrm{XST}+\mathrm{ASA}+\mathrm{CLP}$ cotreated group exhibited more significant increase in $\mathrm{rCBF}$ than the ASA+CLP co-treated group.

\section{$X S T+A S A+C L P$ attenuates neuronal injury}

As shown in Figure 4A, H\&E-stained slides of brain sections 
A
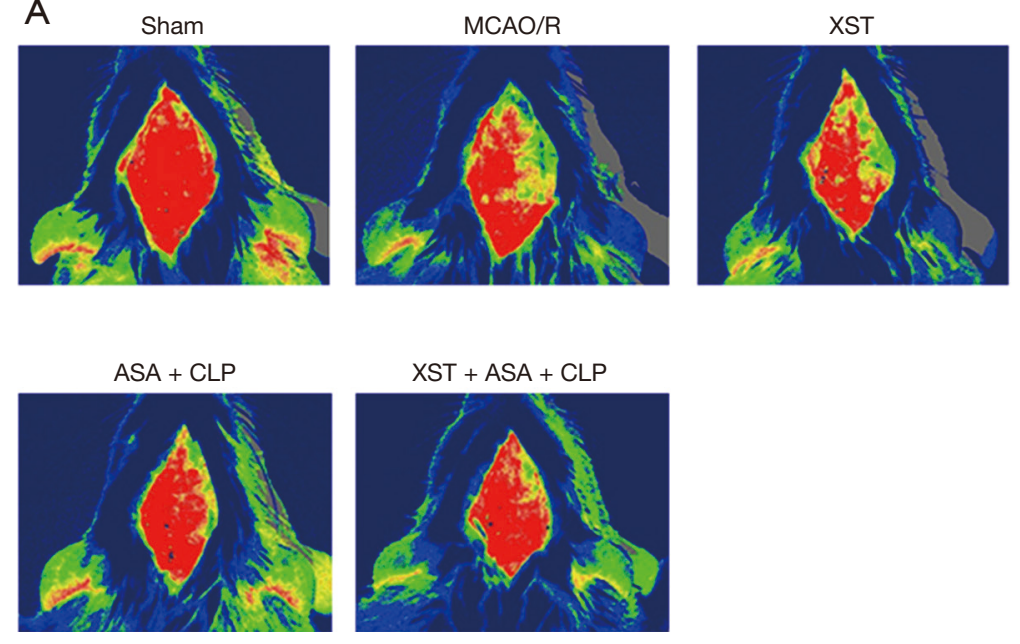

B

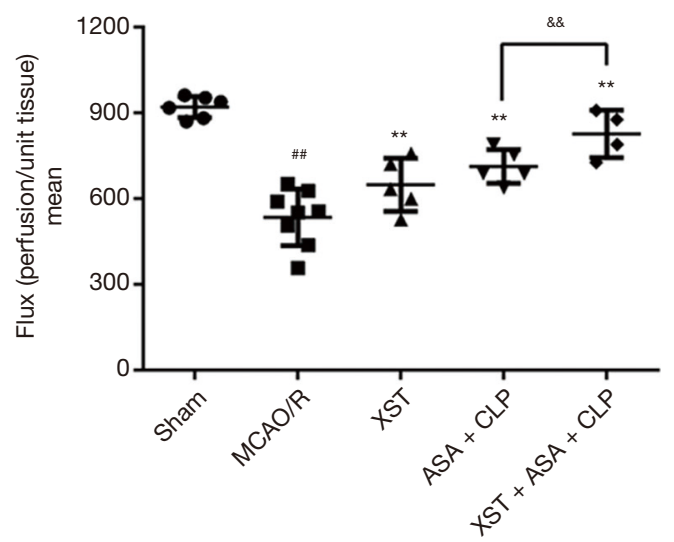

Figure $3 \mathrm{XST}+\mathrm{ASA}+\mathrm{CLP}$ increases regional cerebral blood flow (rCBF) in rats with MCAO/R. (A) The images of rCBF of cortex. The magnitude of $\mathrm{rCBF}$ is represented by different colors, with blue to red indicating low to high. (B) Quantitative analysis of $\mathrm{rCBF}$ in different

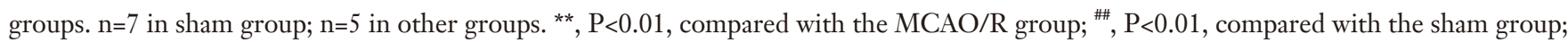
\&\&, $\mathrm{P}<0.01$, compared with the ASA+CLP group. XST, Xuesaitong injection (lyophilized); ASA, aspirin; CLP, clopidogrel; MCAO/R, middle cerebral artery occlusion/reperfusion.

from each group were detected under a light microscope. Untreated rats have many neurons present in the pyknotic nuclei, whereas $\mathrm{MCAO} / \mathrm{R}$ rats have pale nuclei in the hippocampal CA1 regions. Apparently, administration of XST and ASA+CLP notably reduced the pyknotic nuclei in the hippocampal CA1 regions. Apoptotic damage has been implicated in neuronal dysfunction during ischemic stroke. The apoptotic index of brain tissues was assessed to determine whether the observed better neuronal protection of XST+ASA+CLP against I/R-induced neuronal dysfunction was associated with apoptosis (Figure 4B,C). The number of apoptotic neurons was reduced in drugtreated groups compared with MCAO/R rats. Additionally, the neurological performance of XST+ASA+CLP cotreatment rats also dramatically improved compared with ASA+CLP co-treated rats.

\section{$X S T+A S A+C L P$ attenuates activation of microglia and astrocytes}

Microglia and astrocytes have been implicated in neuronal death during ischemic stroke (28). The number of microglia and astrocyte cells positively expressed by Ib $\alpha-1$ and GFAP were assessed to determine whether XST+ASA+CLP has better neuronal protection against I/R-induced neuronal dysfunction than the other treatment regimens
(Figure 5). A larger number of Ib $\alpha-1$-positive cells, namely microglia cells, were observed in the cerebral cortex from $\mathrm{MCAO} / \mathrm{R}$ rats than in those from drug-treated mice $(\mathrm{P}<0.01)$. The number of astrocyte cells that positively expressed GFAP were distinctly increased in the ipsilateral hippocampus of $\mathrm{MCAO} / \mathrm{R}$ rats $(\mathrm{P}<0.05)$. Importantly, attenuated numbers of Ib $\alpha-1$ and GFAP immunoreactivity were discovered in the ipsilateral hippocampus of drugtreated groups $(\mathrm{P}<0.05)$. The XST+ASA+CLP co-treatment had significantly less quantity of Ib $\alpha-1$ than the ASA+CLP co-treated group $(\mathrm{P}<0.05)$.

\section{$X S T+A S A+C L P$ increases neurotrophic factors expression}

Neurotrophic factors, such as brain-derived neurotrophic factor (BDNF), nerve growth factor (NGF), glial cell line-derived neurotrophic factor (GDNF), and vascular endothelial growth factor (VEGF), or insulin-like growth factor 1 (IGF-1), can irritate multiple growth programs and initiate multiple repair processes, including angiogenesis, neurogenesis, and axonal sprouting (29). In the present study, the expression of various growth factors significantly increased in the peri-infarct region after XST+ASA+CLP treatment compared with ASA+CLP treatment (Figure 6). Hence, XST+ASA+CLP stimulated the further production of neurotrophic factors and enhanced neuronal growth. 


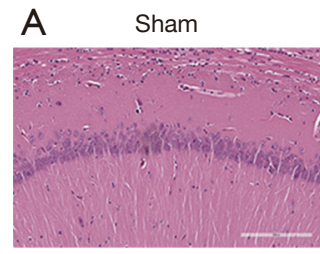

B
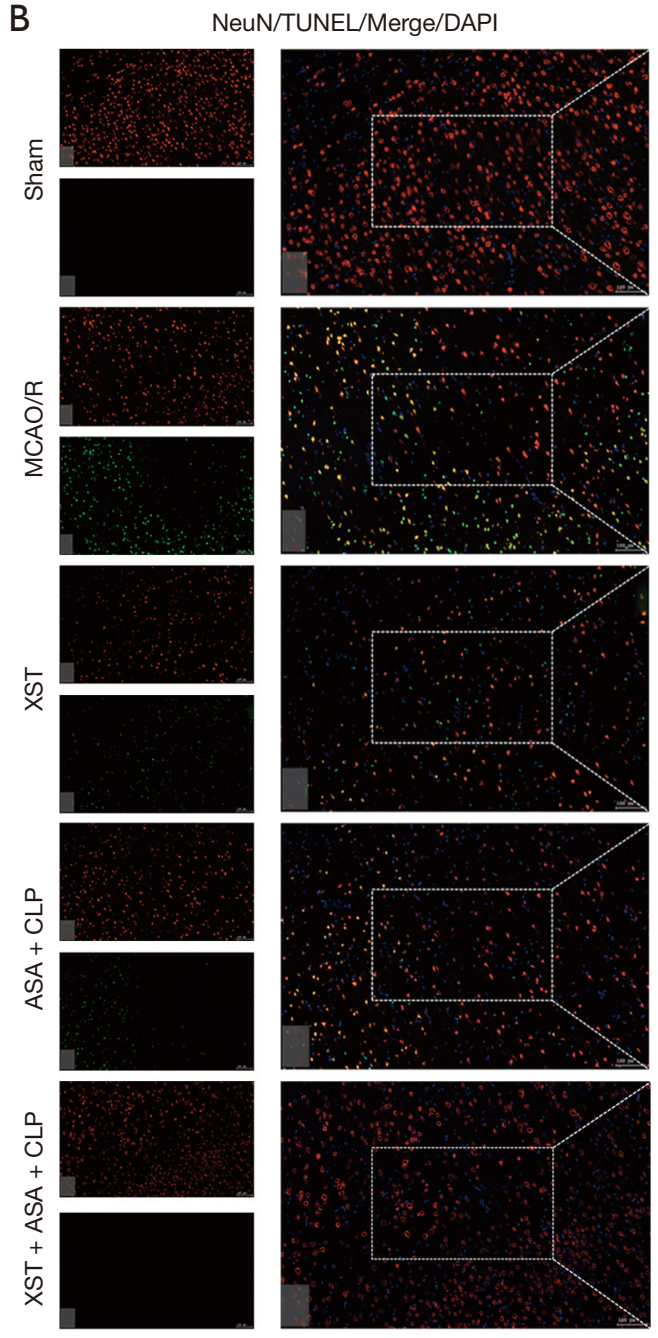

XST
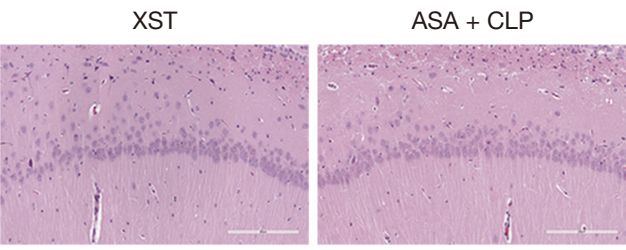

$X S T+A S A+C L P$

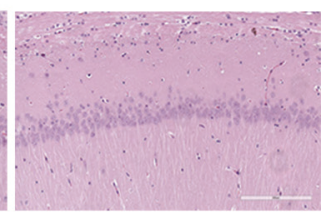

C
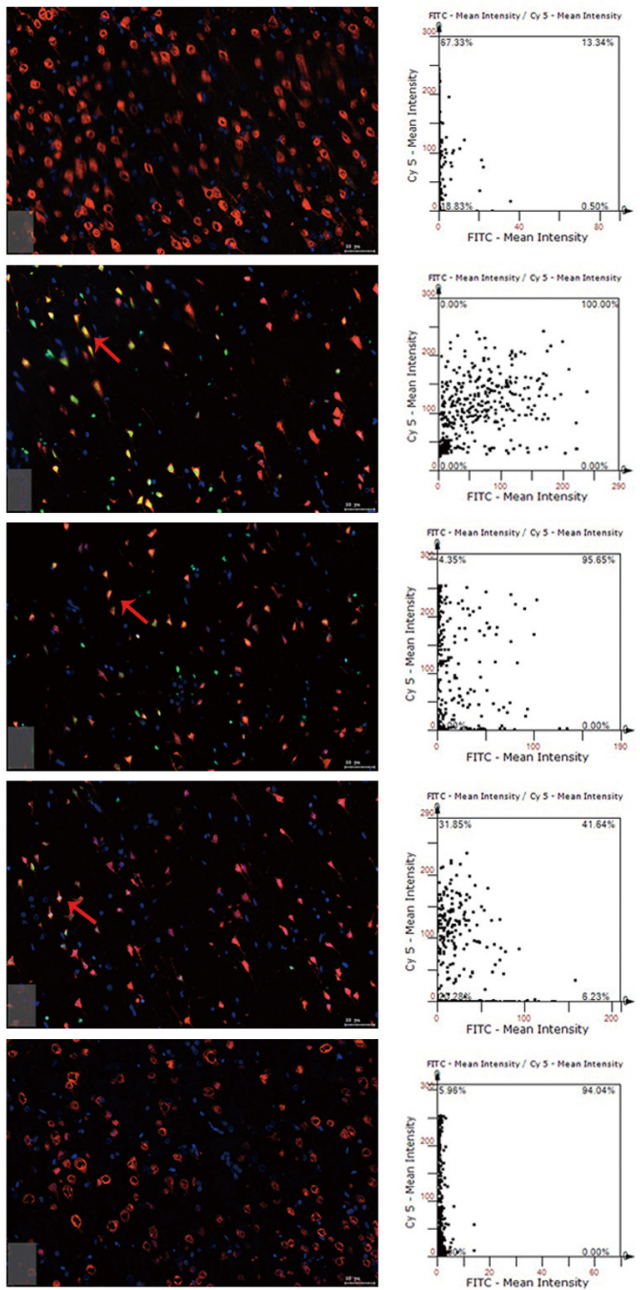

Figure $4 \mathrm{XST}+\mathrm{ASA}+\mathrm{CLP}$ reduces neuronal injury in the hippocampus and cortex area of rats with MCAO/R. (A) H\&E staining in the hippocampal CA1 region in different groups were examined under the light microscope; (B) immunofluorescence for TUNEL-positive neurons in the cortex; (C) quantification of the TUNEL-positive neuron number for different groups. Scale bar: $50 \mu \mathrm{m}$. Values are represented as mean \pm SD for 3 mice in each group. XST, Xuesaitong injection (lyophilized); ASA, aspirin; CLP, clopidogrel; MCAO/R, middle cerebral artery occlusion/reperfusion.

\section{$X S T+A S A+C L P$ alters synaptic function and plasticity}

A comprehensive understanding of the mechanisms of neurotransmitter transmission and synaptic plasticity led us to assess the development of cerebral ischemic symptoms and find potential drug targets for improving them. One of the earliest dysfunctional sites in ischemic stroke is the cortical striatal synapse, in which synapses experience multiple forms of plasticity impairment and neurotransmitter transmission disorders (30). These 
A
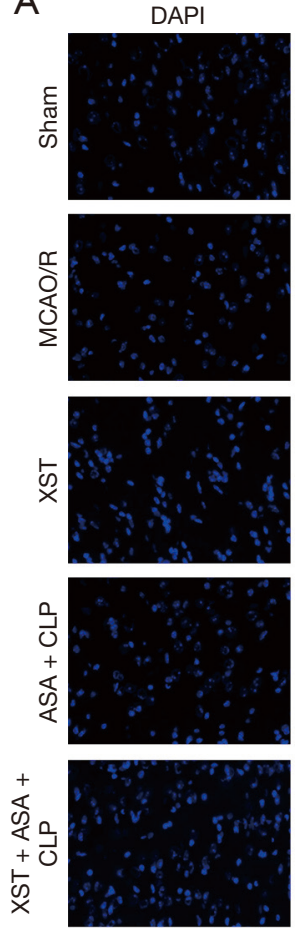

C
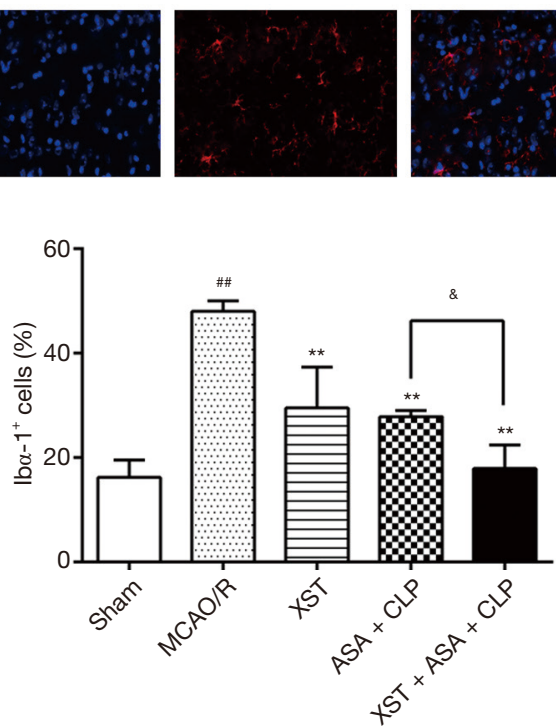

B
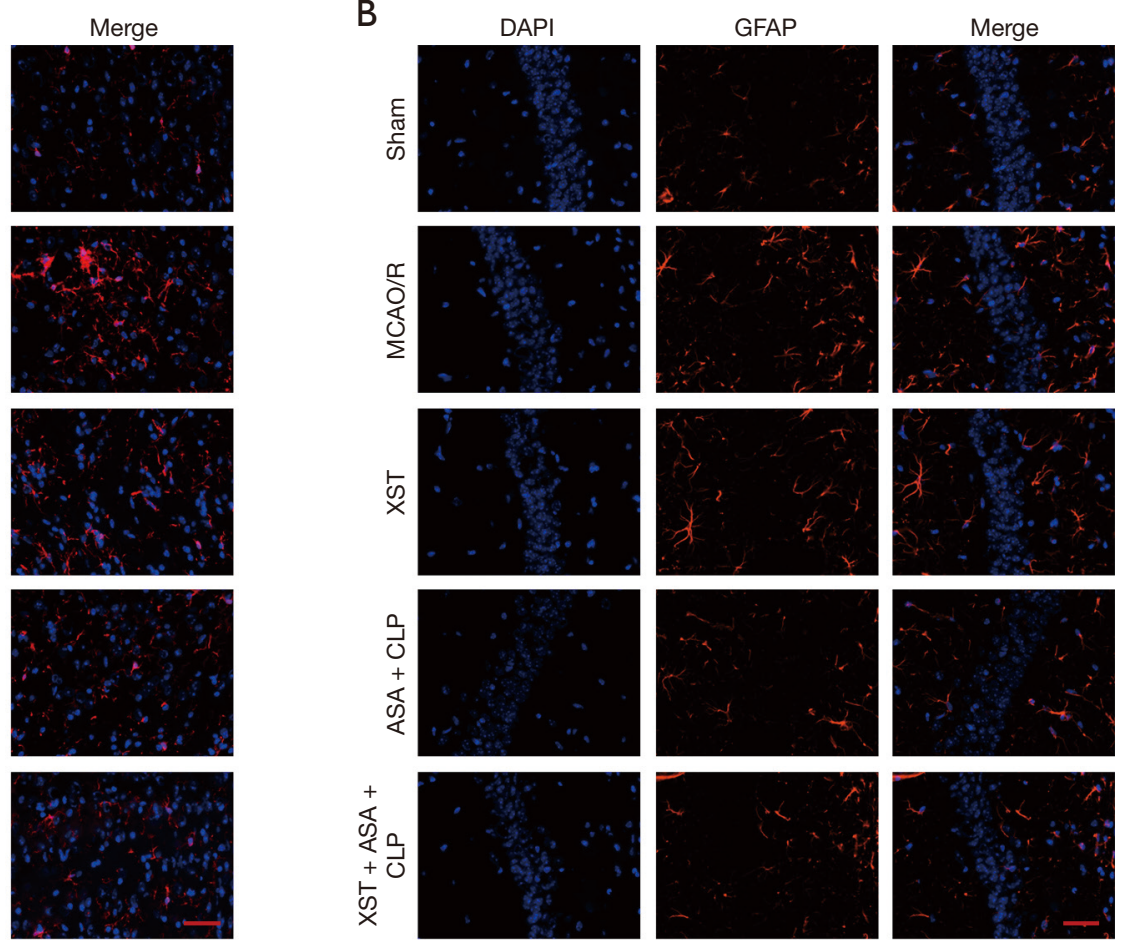

D

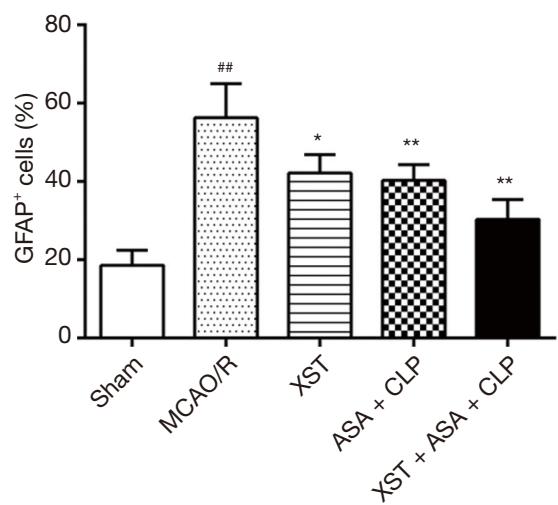

Figure $5 \mathrm{XST}+\mathrm{ASA}+\mathrm{CLP}$ inhibits the activation of microglia and astrocyte in the cortex or hippocampus area of rats with MCAO/R. (A,B) Immunofluorescence for Ib $\alpha-1$ in the cortex and GFAP in the hippocampus. Scale bar: $50 \mu \mathrm{m}$. (C,D) Statistical analysis of total number of $\mathrm{Ib} \alpha-1$ positive cells in the cortex and GFAP positive cells in the hippocampus. Values are represented as means \pm SD for 3 mice in each group. *, $\mathrm{P}<0.05$; **, $\mathrm{P}<0.01$, compared with the MCAO/R group; ${ }^{\# \#}, \mathrm{P}<0.01$, compared with the sham group; ${ }^{*} \mathrm{P}<0.05$, compared with the ASA+CLP group. XST, Xuesaitong injection (lyophilized); ASA, aspirin; CLP, clopidogrel; MCAO/R, middle cerebral artery occlusion/reperfusion.

changes are summarized in Figure 7. XST+ASA+CLP altered some cellular signaling pathways involved in neurotransmitter transmission and synaptic plasticity, which improved neuronal dysfunction. This result helped in understanding the pathogenesis of ischemic stroke, especially early cognitive and subsequent cell death caused by excitotoxicity or loss of nutritional support.

\section{$X S T+A S A+C L P$ inhibits oxidative stress}

During oxidative stress, excess superoxide from NOXs and mitochondria tend to cause lipid peroxidation, DNA oxidation, and protein oxidation. Compared with the sham group, NOX activity and mitochondrial superoxide levels were dramatically increased in the serum of the $\mathrm{MCAO} / \mathrm{R}$ group. The levels of 4-HNE, a marker of 



Figure $6 \mathrm{XST}+\mathrm{ASA}+\mathrm{CLP}$ increases neurotrophic factors expression in rat cortex with MCAO/R. Effect of XST+ASA+CLP treatment on cortex VEGF (A), NGF (B), BDNF (C), GDNF (D) and IGF-1 (E) in rats with MCAO/R. n=6 in sham group; n=8 in other groups. **, $\mathrm{P}<0.01$, compared with the MCAO/R group; ${ }^{\#}, \mathrm{P}<0.05 ;{ }^{\#}, \mathrm{P}<0.01$, compared with the sham group; ${ }^{\&}, \mathrm{P}<0.05 ;{ }^{\& \&}, \mathrm{P}<0.01$, compared with the ASA+CLP group. XST, Xuesaitong injection (lyophilized); ASA, aspirin; CLP, clopidogrel; MCAO/R, middle cerebral artery occlusion/ reperfusion; NGF, nerve growth factor; GDNF, glial cell line-derived neurotrophic factor; VEGF, vascular endothelial growth factor.

lipid peroxidation; protein carbonyl, a marker of protein oxidation; and 8-OHdG, a marker of DNA oxidation products in the serum of rats were remarkably higher in the $\mathrm{MCAO} / \mathrm{R}$ group $(\mathrm{P}<0.01)$. However, 4-HNE, protein carbonyl, and 8-OHdG levels of the drug-treated groups were significantly lower than those of the $\mathrm{MCAO} / \mathrm{R}$ group $(\mathrm{P}<0.01)$. Moreover, NOX and $8-\mathrm{OHdG}$ levels were significantly lower in the XST+ASA+CLP co-treatment group than in the ASA+CLP co-treatment group (Figure 8) $(\mathrm{P}<0.01)$.

\section{$X S T+A S A+C L P$ inhibits inflammatory factors expression}

The protein expression levels of TNF- $\alpha$, IL-1 $\beta$, IL-6, ICAM-1, CD11a, MCP-1, IL-10, and IL-4 in the hippocampus and cortex were not significantly different between MCAO/R-treated and XST+ASA+CLP co-treated mice (Figures 9, Figure S1). However, the expression levels of IL-6, ICAM-1, CD11a, and MCP-1 in the hippocampus decreased in $\mathrm{XST}+\mathrm{ASA}+\mathrm{CLP}$ co-treated mice compared with those of ASA+CLP co-treated mice $(\mathrm{P}<0.05)$. The expression levels of IL-10 and IL-4 in the hippocampus significantly increased in XST+ASA+CLP co-treated mice compared with ASA+CLP co-treated mice $(\mathrm{P}<0.05)$. This phenomenon in cortex IL-6, CD11a, MCP-1, and IL-10 was significantly changed in XST+ASA+CLP co-treated mice compared with that in ASA+CLP co-treated mice $(\mathrm{P}>0.05)$. Hence, $\mathrm{XST}+\mathrm{ASA}+\mathrm{CLP}$ pretreatment leads to the suppression of brain inflammatory responses during ischemic stroke.

The effects of XST+ASA+CLP co-treated mice on $\mathrm{MCAO} / \mathrm{R}$-induced inflammatory response were also detected by the serum levels of circulating inflammatory cytokines (Figure S2). The expression levels of TNF- $\alpha$, IL-1 $\beta$, IL-6, ICAM-1, CD11a, and MCP-1 decreased in rat serum after MCAO/R. By contrast, XST+ASA+CLP 
A

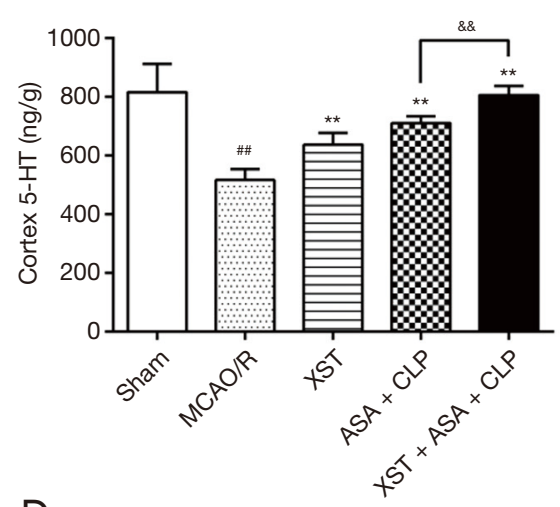

$\mathrm{D}$

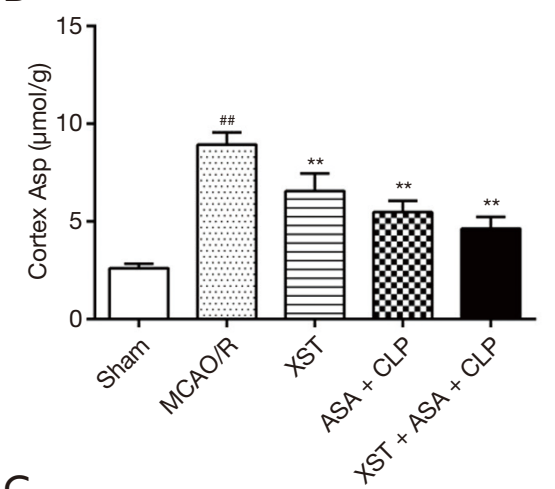

G

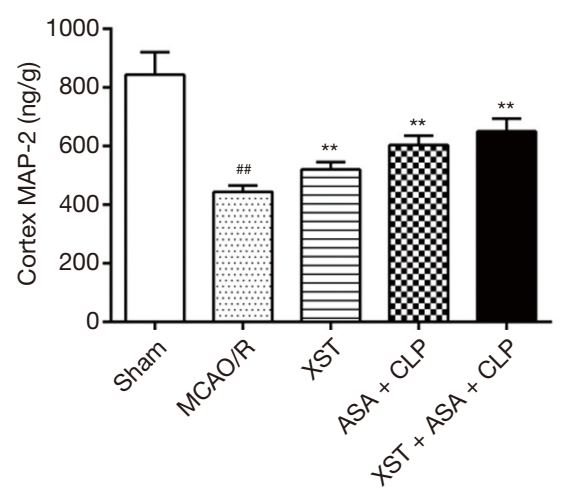

B

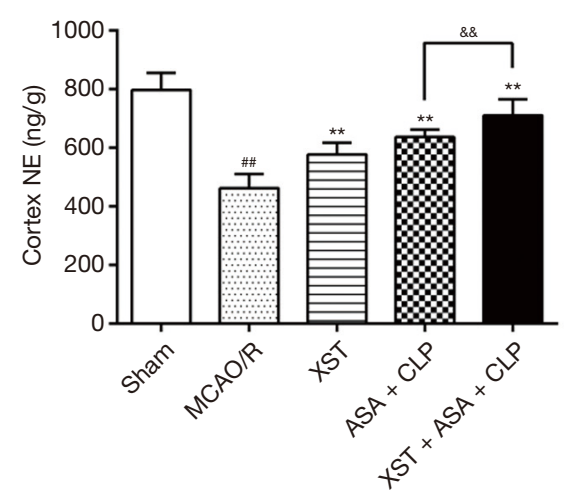

E

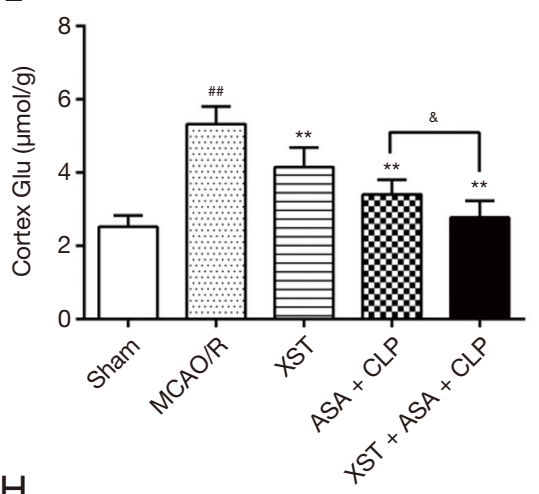

$\mathrm{H}$

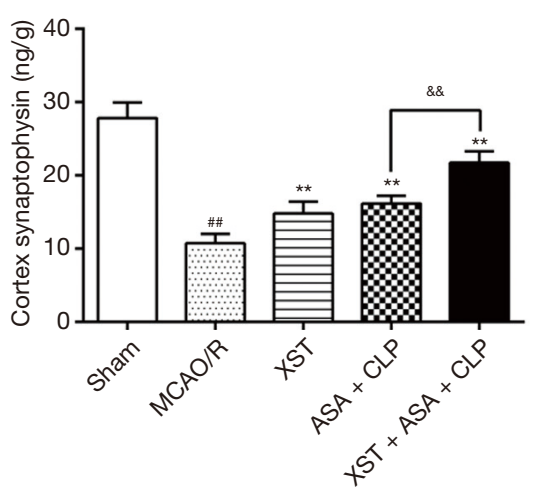

C

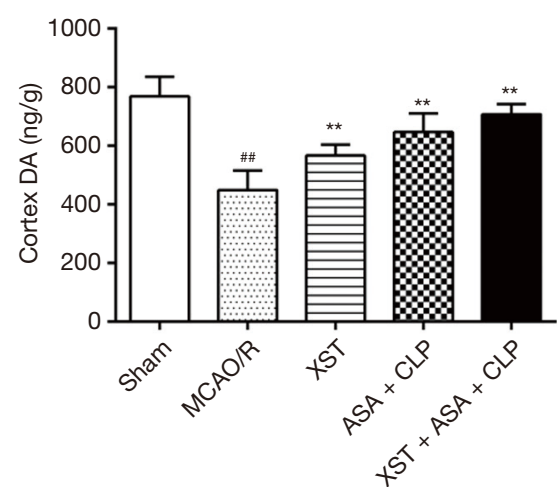

$\mathrm{F}$

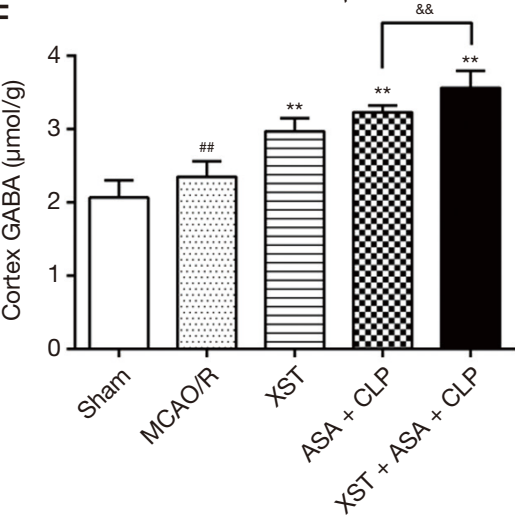

Figure $7 \mathrm{XST}+\mathrm{ASA}+\mathrm{CLP}$ alters synaptic function and plasticity in rats cortex with MCAO/R. Effect of XST+ASA+CLP treatment on cortex 5-HT (A), NE (B), DA (C), Asp (D), Glu (E), GABA (F), MAP-2 (G) and synaptophysin (H) in rats with MCAO/R. n=6 in sham group; $\mathrm{n}=8$ in other groups. ${ }^{* *}, \mathrm{P}<0.01$, compared with the $\mathrm{MCAO} / \mathrm{R}$ group; ${ }^{*}, \mathrm{P}<0.01$, compared with the sham group; ${ }^{*}, \mathrm{P}<0.05 ;{ }^{\text {\& } \&}$, $\mathrm{P}<0.01$, compared with the ASA+CLP group. XST, Xuesaitong injection (lyophilized); ASA, aspirin; CLP, clopidogrel; MCAO/R, middle cerebral artery occlusion/reperfusion; 5-HT, 5-hydroxytryptamine; NE, norepinephrine; DA, dopamine; Asp, aspartate; Glu, glutamate; GABA, gamma-aminobutyric acid; MAP-2, microtubule associated protein-2.

pretreatment significantly inhibited the decrease in the serum levels of TNF- $\alpha$, IL-1 $\beta$, IL-6, ICAM-1, CD11a, and MCP-1 caused by MCAO/R. We also measured a significant stimulatory effect of XST+ASA+CLP on the levels of IL-10 and IL-4 in the serum of MCAO/R-treated rats.

\section{$X S T+A S A+C L P$ suppresses NOX2 expression and IL-6/ STAT3 patbway}

The expression levels of NOX2, iNOS, p-STAT3, STAT3, and IL-6 in MCAO/R-treated rats cortex tissues were 

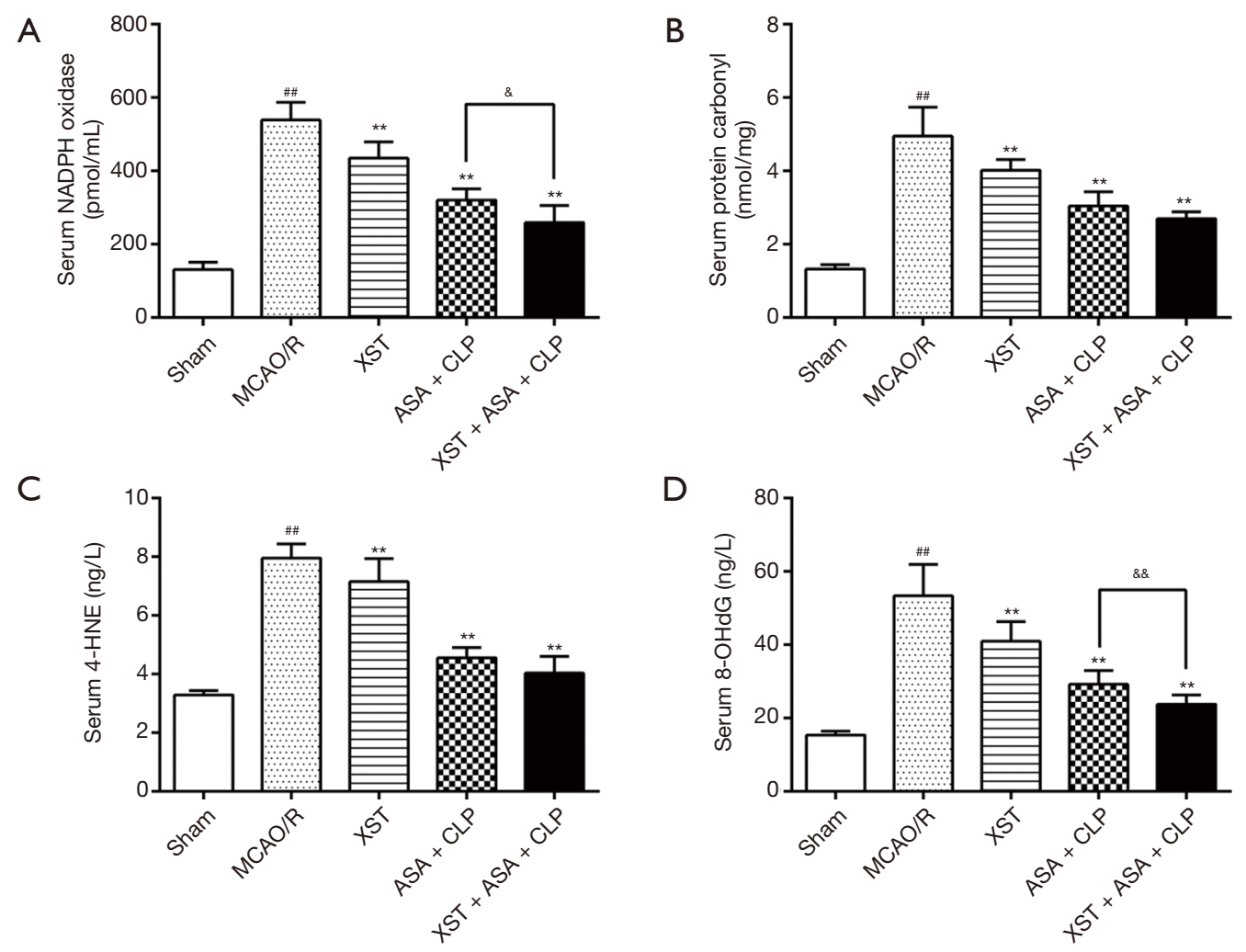

Figure $8 \mathrm{XST}+\mathrm{ASA}+\mathrm{CLP}$ inhibits oxidative stress in rat serum with MCAO/R. Effect of XST+ASA+CLP treatment on serum NADPH (A), protein carbonyl (B), 4-HNE (C) and 8-OHdG (D) in rats with $\mathrm{MCAO} / \mathrm{R} . \mathrm{n}=6$ in sham group; $\mathrm{n}=8$ in other groups. **, $\mathrm{P}<0.01$, compared with the MCAO/R group; ${ }^{\#}, \mathrm{P}<0.01$, compared with the sham group; ${ }^{*}, \mathrm{P}<0.05 ;{ }^{\& \&}, \mathrm{P}<0.01$, compared with the ASA+CLP group. XST, Xuesaitong injection (lyophilized); ASA, aspirin; CLP, clopidogrel; MCAO/R, middle cerebral artery occlusion/reperfusion; NADPH, nicotinamide adenosine dinucleotide phosphate; 4-HNE, 4-hydroxynonenal; 8-OHdG, 8-hydroxydeoxyguanosine.

measured using Western blot analysis to understand the mechanism of XST+ASA+CLP on ischemic stroke protection (Figure 10). The Western blot results revealed that the XST, ASA+CLP, and XST+ASA+CLP treatments significantly reduced the expression levels of NOX2, iNOS, p-STAT3/STAT3, and IL-6 in the ischemic cortex compared with the $\mathrm{MCAO} / \mathrm{R}$ group $(\mathrm{P}<0.01)$. XST+ASA+CLP treatment remarkably upregulated the expression of $\mathrm{p}$-STAT3/STAT3 compared with the ASA+CLP treatment $(\mathrm{P}<0.01)$.

\section{Discussion}

In this study, $\mathrm{MCAO} / \mathrm{R}$ rat model was used to investigate the effects and possible mechanisms of XST+ASA+CLP following acute ischemic stroke through the suppression of the NOX2/IL-6/STAT-3 pathway. Results show that infarct volume, neurological deficit, rCBF, blood- brain barrier (BBB) permeability (Figure S3), synaptic function and plasticity, neuronal injury, and glial activation were improved in the model by XST, ASA+CLP, and XST+ASA+CLP pretreatment. Additionally, the pretreated rats exhibited decreased NOX, protein carbonyl, 4-HNE, $8-\mathrm{OHdG}$, and the activity of various inflammatory factors compared with the MCAO/R group. Furthermore, $\mathrm{XST}+\mathrm{ASA}+\mathrm{CLP}$ treatment has more effectively protected $\mathrm{MCAO} / \mathrm{R}$ rats than the XST and ASA+CLP treatment. Hence, XST+ASA+CLP could be a new clinical ischemic stroke therapy.

DAPT with ASA and CLP was more frequently applied to patients with different risk factors, placing them at rising risk of recurrent stroke, including prior ischemic events, concomitant vascular risk factors, and index events present in antiplatelet therapy (31). Although DAPT with ASA and CLP co-therapy in early recurrent stroke and coronary artery disease were widely acknowledged, joint use of 
A
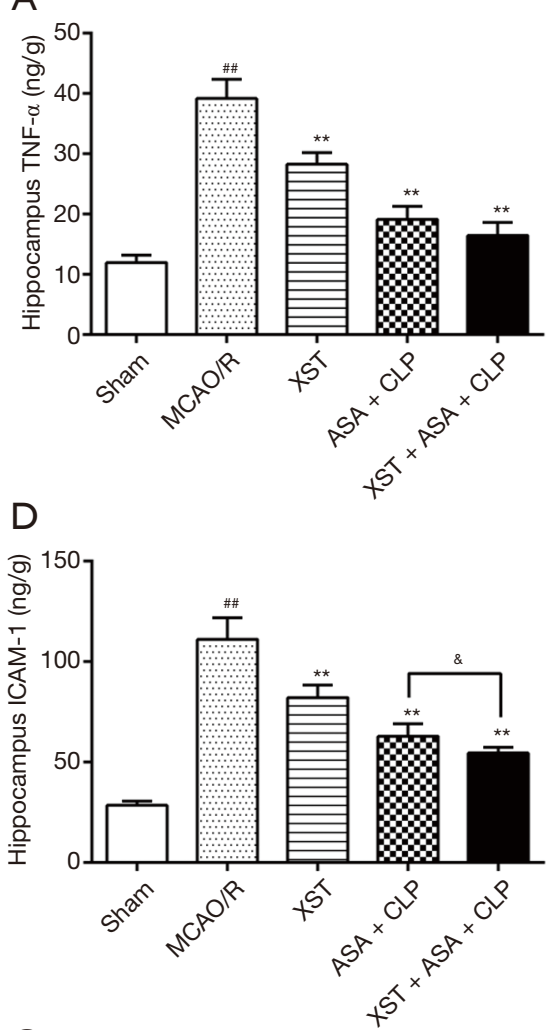

G

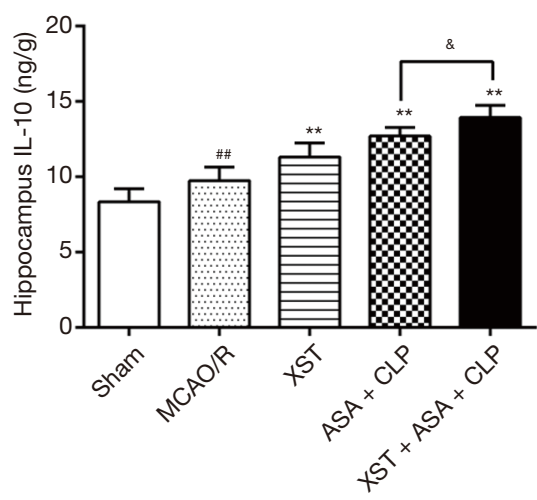

B

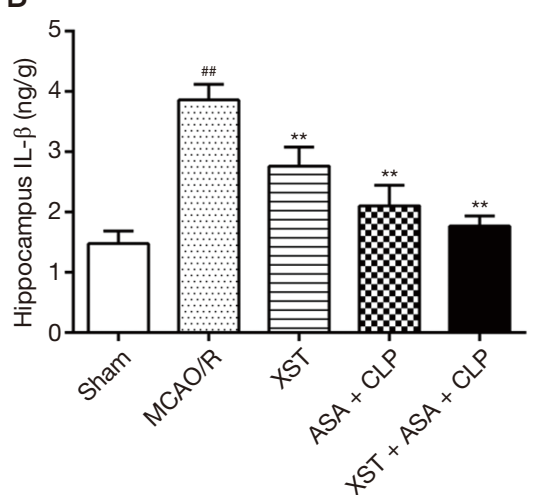

E

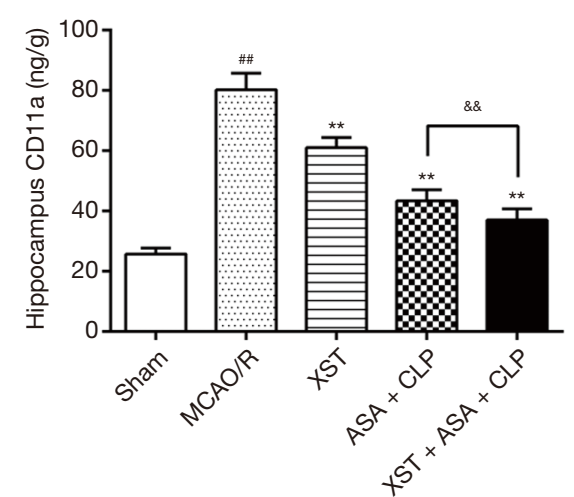

$\mathrm{H}$

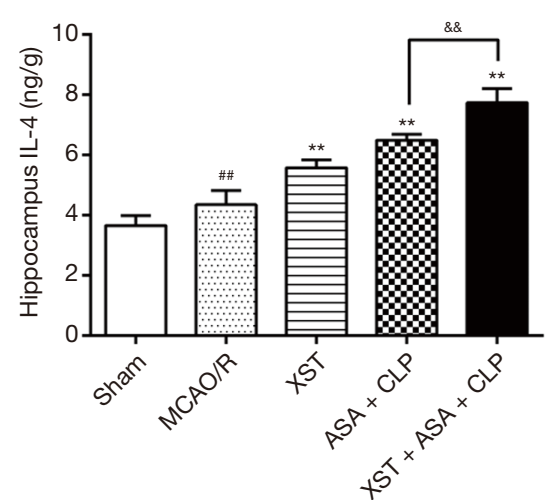

C

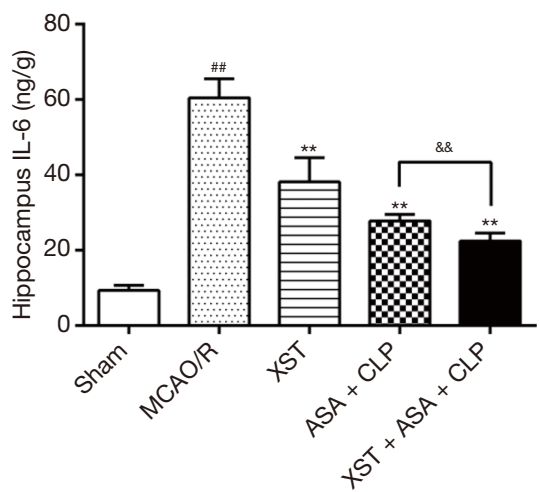

$\mathrm{F}$

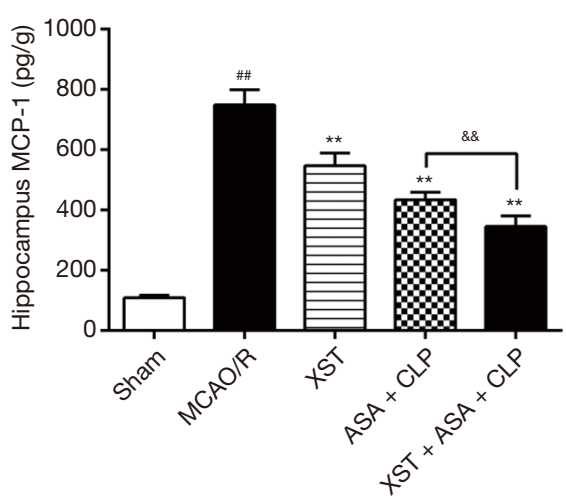

Figure $9 \mathrm{XST}+\mathrm{ASA}+\mathrm{CLP}$ inhibits inflammatory response in rats hippocampus with MCAO/R. Effect of XST+ASA+CLP treatment on hippocampus TNF- $\alpha$ (A), IL-1 $\beta$ (B), IL-6 (C), ICAM-1 (D), CD11a (E), MCP-1 (F), IL-10 (G) and IL-4 (H) in rats with MCAO/R. n=6 in sham group; $\mathrm{n}=8$ in other groups. ${ }^{* *}, \mathrm{P}<0.01$, compared with the $\mathrm{MCAO} / \mathrm{R}$ group; ${ }^{\# \#,} \mathrm{P}<0.01$, compared with the sham group; ${ }^{*}, \mathrm{P}<0.05$; \&\&, $\mathrm{P}<0.01$, compared with the ASA+CLP group. XST, Xuesaitong injection (lyophilized); ASA, aspirin; CLP, clopidogrel; MCAO/R, middle cerebral artery occlusion/reperfusion; TNF- $\alpha$, tumor necrosis factor-alpha; IL-1 $\beta$, interleukin-1 beta; IL-6, interleukin-6; ICAM-1, intercellular adhesion molecule-1; MCP-1, monocyte chemoattractant protein-1; IL-10, interleukin-10; IL-4, interleukin-4.

Chinese and Western therapy has been gaining popularity in the recent years. However, the related medicine interactions between DAPT and traditional Chinese medicine were barely known. XST is a freeze-dried saponin powder injection of $P$. notoginseng saponins, which has been widely used in China for preventing and treating cerebral ischemic stroke (28). XST has a strong neuroprotective function against oxidative stress and inflammation $(32,33)$. 
A

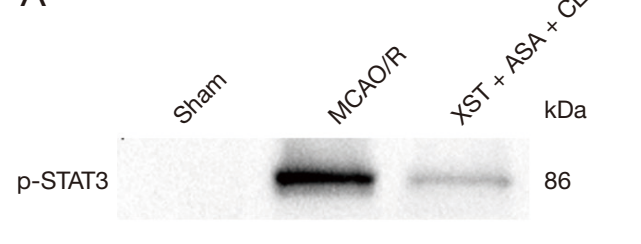

STAT3

86
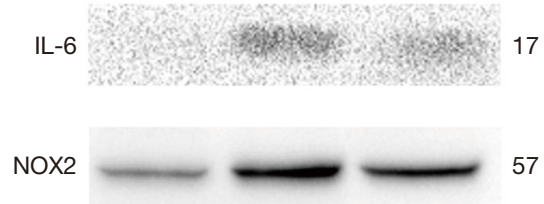

iNOS

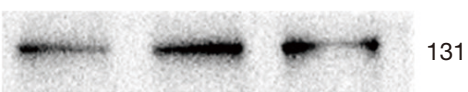

$\beta$-actin

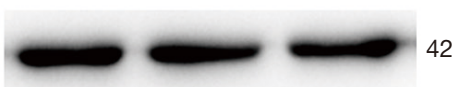

B
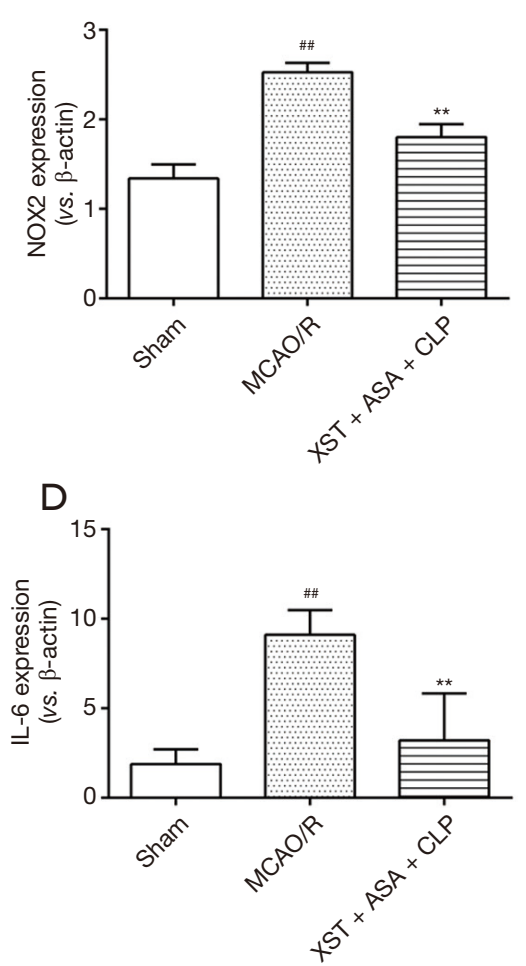

C

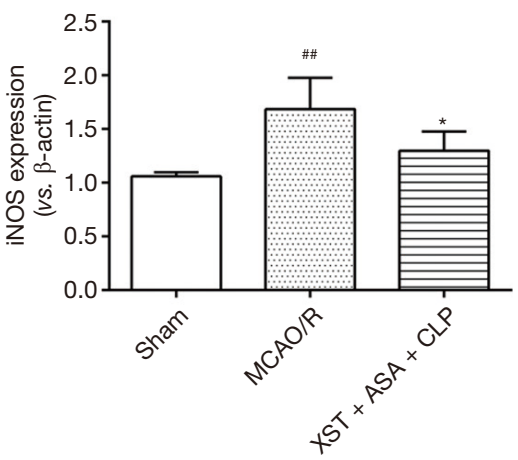

E

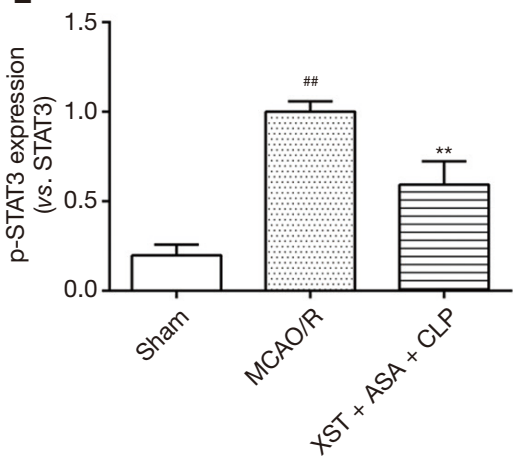

Figure $10 \mathrm{XST}+\mathrm{ASA}+\mathrm{CLP}$ inhibits the expression of NOX2 and IL-6/STAT-3 signaling after ischemic stroke. (A) Protein expressions of p-STAT3, STAT3, IL-6, NOX2 and iNOS were measured with Western blot; (B) relative protein expression level to $\beta$-actin ratios were evaluated. Values are represented as means $\pm \mathrm{SD}$ for 3 mice in each group. ${ }^{*}, \mathrm{P}<0.05 ;{ }^{* *}, \mathrm{P}<0.01$, compared with the MCAO/R group; ${ }^{\# \#}$ $\mathrm{P}<0.01$, compared with the Sham group. XST, Xuesaitong injection (lyophilized); ASA, aspirin; CLP, clopidogrel; MCAO/R, middle cerebral artery occlusion/reperfusion; NOX2, nicotinamide adenosine dinucleotide phosphate oxidase 2; IL-6, interleukin-6; iNOS, inducible nitric oxide synthase.

Aside from studies that reported the inhibition effect of XST monotherapy on oxidative stress and inflammation, there is no study on the effect of XST and DAPT cotherapy protecting against ischemic stroke against MCAO/ $\mathrm{R}$ rats by inhibiting oxidative stress and inflammation. Based on the current pharmacological findings, the antioxidative and anti-inflammatory effects of XST, ASA+CLP, and $\mathrm{XST}+\mathrm{ASA}+\mathrm{CLP}$ in cerebral $\mathrm{MCAO} / \mathrm{R}$ injuries were compared, and the relative potential mechanisms of the neuroprotection of XST+ASA+CLP were suggested. More importantly, joint use strategy was investigated to determine whether the drugs could compensate for their individual weaknesses to treat ischemic stroke, or have better outcomes than the monotherapy of drugs, and designed XST and $\mathrm{XST}+\mathrm{ASA}+\mathrm{CLP}$ groups.

Reactive gliosis as a result of ischemic stroke involves astrocytes and microglia, which are pivotal components of cellular and molecular pathways involved in stroke-induced brain injury (34). The present data confirmed that MCAO/ $\mathrm{R}$ injury elevated the expression levels of GFAP and Ib $\alpha-1$, whereas the expression of these markers decreased in rats pretreated with XST, ASA+CLP, and XST+ASA+CLP. This phenomenon indicates that the neuroprotective effects of XST, ASA+CLP, and XST+ASA+CLP were attributed to the inhibition of the proliferation of glial cells.

Changes in neuronal activity observed in neurological disorders are associated with many neurotransmitters, including synaptic activity and neuronal plasticity. Neurite outgrowth is a key morphological feature that characterizes neuronal development and an important aspect of neural regeneration that determines neuronal plasticity and neuropathological conditions (35). In the present study, a series of neurochemical mediators and modulators of synaptic transmission were destroyed in 
ischemic stroke. Classical neurotransmitters like gammaaminobutyric acid (GABA), glutamate (Glu), and aspartic acid (Asp); modulators such as 5-hydroxytryptamine (5-HT), dopamine (DA), and norepinephrine (NE); and molecules like BDNF, which indirectly regulates neurotransmission, were destroyed in ischemic stroke. Here, XST with DAPT facilitates the properties of neurotrophic factors. These neurotrophic factors, especially BDNF, promoted synaptic activity and plasticity after stroke and stimulated the proliferation of 5-HT, NE, DA, GABA, microtubule associated protein-2 (MAP-2), and synaptophysin and the downregulation of Asp and Glu. These effects may promote neuronal growth and survival in the developing CNS.

Excessive oxidative stress plays a crucial role in the progression of acute brain injury in both human and animal studies (36). At the molecular level, oxidative stress exacerbates the inflammatory response (37) and triggers the activation of matrix metalloproteinases (38), thereby disrupting the integrity of $\mathrm{BBB}$ and accelerating neuronal apoptosis and white matter damage. Basic scientific research on oxidative stress is necessary when studying a prospective intervention in stroke pathology studies (39). Oxidative stress during cerebral MCAO/R injury is characterized by high concentration of ROS, which rapidly overwhelms endogenous antioxidant defense. Intracellular ROS causes oxidation of lipids, proteins, and DNA and alters the final link that promotes neuronal apoptosis signaling pathways (40). Neuronal cells are more likely affected by ROS due to their higher oxidative metabolism and membrane fatty acid content and less antioxidant enzymes (41). Inhibiting the source of ROS is a novel strategy for the treatment of acute ischemic stroke (42). Superoxide is the first ROS produced in the oxygen free radical chain in the early stages of reperfusion. NOX is a direct source of superoxide $(7,10)$ and is activated by a variety of stimuli including angiotensin II, NE, and tumor necrosis factor (43-46), which are related to the progression of neurodegenerative and cardiovascular diseases (47). Increased expression of NOX in rats is related to aggravated ischemic stroke (48). NOXmutant mice treated with $\mathrm{MCAO} / \mathrm{R}$ exhibited ameliorative neurobehavioral effect and reduced infarct size (49). NOX isoform NOX2 deletion and superoxide dismutase 2 elevation contributes to neuroprotective effects against memory impairment and hippocampal damage (45). NOX2 is activated and triggers the development of oxidative stressrelated damage after subarachnoid hemorrhage (50). The present in vitro study has shown that XST, ASA+CLP, and $\mathrm{XST}+\mathrm{ASA}+\mathrm{CLP}$ pretreatments inhibited the increased levels of oxidative stress markers (such as NADPH, protein carbonyl, 4-HNE, and 8-OHdG) induced by $\mathrm{MCAO} / \mathrm{R}$ in rats. Moreover, NADPH and 8-OHdG levels significantly decreased in MCAO/R rats co-treated with ASA, CLP, and XST compared with those treated with XST alone and ASA+CLP. These results showed that the neuroprotective effect of XST+ASA+CLP were partly caused by oxidative stress inhibition.

Microglia activation and acute neuronal cell death after $\mathrm{MCAO} / \mathrm{R}$ injury are related to inflammatory mechanisms $(34,51)$. During this pathological process, inflammatory cascades are triggered by energy depletion and necrotic neuron death in the ischemic region. Therefore, inhibition of pro-inflammatory cytokines, particularly TNF- $\alpha$, IL- $1 \beta$, IL-6, ICAM-1, CD11a, and MCP-1, are likely to have neuroprotective effects during $\mathrm{MCAO} / \mathrm{R}$ injury. The present study showed that MCAO/R significantly deteriorated neurological function and facilitated microglia activation and neuronal cell apoptosis in the brain. These phenomena were accompanied by the enhanced expression of a series of pro-inflammatory cytokines such as TNF- $\alpha$, IL- $1 \beta$, IL-6, ICAM-1, CD11a, and MCP-1. In contrast, XST, ASA+CLP, and $\mathrm{XST}+\mathrm{ASA}+\mathrm{CLP}$ inhibited all above-mentioned activities induced by MCAO/R challenge. The results also show that the release of anti-inflammatory IL-4 and IL-10 was elevated by XST, ASA+CLP, and XST+ASA+CLP pretreatment. IL-10 attenuates inflammatory milieu in the CNS, inhibits microglia activation, maintains $\mathrm{BBB}$ integrity, and lessens neurodegeneration (52). The increase in protein levels of ICAM-1 measured from the serum, cortex, and hippocampus tissues following $\mathrm{MCAO} / \mathrm{R}$ challenge is consistent with a previous research manifesting that high ICAM-1 levels were more likely to have previous stroke and increased hemorrhagic transformation risk (53). This increase was triggered by $\mathrm{MCAO} / \mathrm{R}$ challenge and inflammatory cytokine overproduction, which was dramatically limited by XST, ASA+CLP, and $\mathrm{XST}+\mathrm{ASA}+\mathrm{CLP}$ pretreatments. Moreover, inflammatory cytokine levels significantly decreased in $\mathrm{MCAO} / \mathrm{R}$ rats co-treated with ASA, CLP, and XST compared with those treated with XST alone and ASA+CLP.

To explore the mechanism by which XST+ASA+CLP had better moderating effect on oxidative stress after MCAO/ $\mathrm{R}$ challenge, we discussed the effects of XST+ASA+CLP on NOX2 expression after MCAO/R challenge. Present results revealed that NOX2 expression was dramatically increased after MCAO/R challenge. Previous results have revealed that NOX2 transforms microglia from a pro-inflammatory 


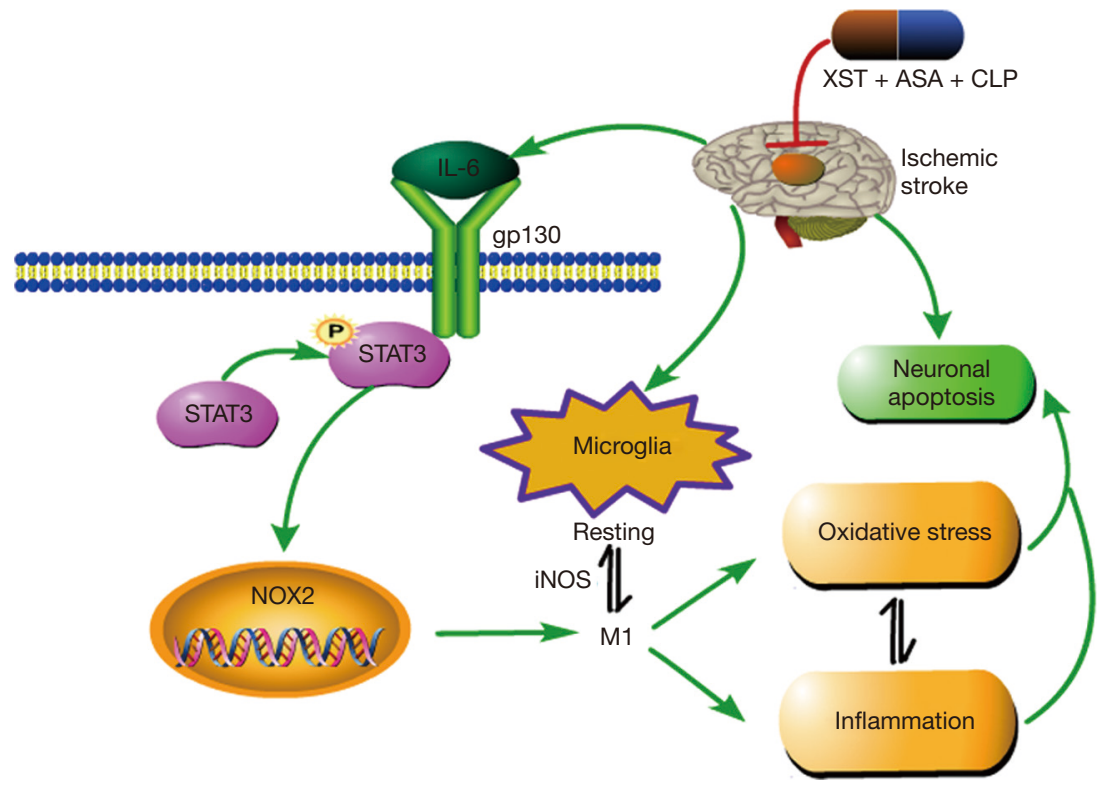

Figure 11 Scheme of mechanisms underlying protective property of ischemic stroke induced by MCAO/R, after XST+ASA+CLP treatment. IL-6/STAT3 pathway increases NOX2 expression in ischemic stage can lead to transform resting microglia to M1 microglia, result in oxidative stress and inflammation. Ultimately, neuronal apoptosis. XST+ASA+CLP suppresses the activation of IL-6/STAT3/NOX2 axis induced by ischemic stroke in rats. XST, Xuesaitong injection (lyophilized); ASA, aspirin; CLP, clopidogrel; MCAO/R, middle cerebral artery occlusion/reperfusion; NOX2, nicotinamide adenosine dinucleotide phosphate oxidase 2; IL-6, interleukin-6; iNOS, inducible nitric oxide synthase.

M1 phenotype to an anti-inflammatory M2 phenotype after traumatic brain injury, and that the weakening of NOX2 inhibits M1 microglia and improves brain damage (54). Interestingly, this previous study reported that microglia, which exhibit pro-inflammatory phenotype, was connected with oxidative stress and pro-inflammatory cytokine expression, leading to the onset of a vicious circle and ultimately to more severe brain damage (Figure 11). In the present study, MCAO/R significantly increased pro-inflammatory microglia and pro-inflammatory cytokines TNF- $\alpha$, IL-1 $\beta$, and IL-6 expression in MCAO/ R-treated group compared with Sham group. By contrast, $\mathrm{XST}+\mathrm{ASA}+\mathrm{CLP}$ pretreatment remarkably reduced NOX2 expression and reversed inflammatory microglia activation by inhibiting oxidative stress marker and pro-inflammatory cytokines after ischemic stroke.

The IL-6/STAT3 signaling pathway acts as signal transduction in a series of brain injuries, in which STAT3 is a key factor in NOX2 activation $(36,55)$ and can induce pro-inflammatory immune reaction, such as IL-6 (56), which is an important cause of several brain damage activation $(57,58)$. The complex formed by the binding of IL-6 to the soluble IL-6 receptor is associated with the membrane glycoprotein gp130, which activates JAK phosphorylation coupled to gp130, resulting in STAT3 phosphorylation and leading to the onset and persistence of inflammation (59). NOX2 upstream regulator, the IL-6/ STAT3 signaling pathway, was detected to determine how the XST+ASA+CLP treatment inhibits M1 microglia activation after MCAO/R. The present results showed that $\mathrm{XST}+\mathrm{ASA}+\mathrm{CLP}$ pretreatment diminished the expression of phosphorylation STAT3 and IL-6 in vivo, as shown by analysis using Western blot. The results suggest that $\mathrm{XST}+\mathrm{ASA}+\mathrm{CLP}$ downregulated NOX2 and that this downregulation is accompanied by the IL-6/STAT3 signaling pathway.

This study also has some limitations. We used an acute rather than a chronic cerebral ischemia model, as the duration of ischemia was 2 hours. Therefore, a long-term cerebral ischemic experiment may have different effects on oxidative stress and inflammation. Furthermore, the signaling pathways associated with oxidative stress and inflammation involve not only NOX2 and IL-6/STAT3 pathways, but also several other mediators that have not 
been evaluated in our study, such as Nrf2 and NLRP3. Finally, clinical not just animal experiments should be conducted for a more comprehensive pharmacodynamic evaluation and mechanistic study.

\section{Conclusions}

In summary, XST+ASA+CLP showed more neuroprotective activity against oxidative stress and inflammation induced by $\mathrm{MCAO} / \mathrm{R}$ in rats. These neuroprotective effects were attributed to restrain superoxide formation by inhibiting NOX2 activity. The neuroprotective mechanisms of $\mathrm{XST}+\mathrm{ASA}+\mathrm{CLP}$ involved the inhibition of NOX2 and IL-6/STAT3 signaling pathway after ischemic stroke.

\section{Acknowledgments}

Funding: This study was supported by the National Natural Science Foundation of China (No. 81891012), the Drug Innovation Major Project (No. 2018ZX09711001-009), the National Key R\&D Plan (No. 2017YFC1702504), Central Public Interest Scientific Institution Basal Research Fund (No. 2018PT35030) and the National Key R\&D Plan (No. 2018YFC1707408).

\section{Footnote}

Reporting Checklist: The authors have completed the ARRIVE reporting checklist. Available at http://dx.doi. org/10.21037/apm-20-1681

Data Sharing Statement: Available at http://dx.doi. org/10.21037/apm-20-1681

Conflicts of Interest: All authors have completed the ICMJE uniform disclosure form (available at http://dx.doi. org/10.21037/apm-20-1681). The authors have no conflicts of interest to declare.

Ethical Statement: The authors are accountable for all aspects of the work in ensuring that questions related to the accuracy or integrity of any part of the work are appropriately investigated and resolved. All animal care and experimental procedures were reported in accordance with the Institutional Animal Care and Use Committee of the Chinese Academy of Medical Sciences \& Peking Union Medical College and complied with NIH Guidelines for the Care and Use of Laboratory Animals (approval number:
SYXK 2017-0020). All efforts were followed to reduce the number of animals used and ensure minimal suffering.

Open Access Statement: This is an Open Access article distributed in accordance with the Creative Commons Attribution-NonCommercial-NoDerivs 4.0 International License (CC BY-NC-ND 4.0), which permits the noncommercial replication and distribution of the article with the strict proviso that no changes or edits are made and the original work is properly cited (including links to both the formal publication through the relevant DOI and the license). See: https://creativecommons.org/licenses/by-nc-nd/4.0/.

\section{References}

1. Mozaffarian D, Benjamin EJ, Go AS, et al. Heart Disease and Stroke Statistics-2016 Update: A Report from the American Heart Association. Circulation 2016;133:e38360.

2. Zhou Z, Lu J, Liu WW, et al. Advances in stroke pharmacology. Pharmacol Ther 2018;191:23-42.

3. Iadecola $\mathrm{C}$ and Anrather J. The immunology of stroke: from mechanisms to translation. Nat Med 2011;17:796-808.

4. Ransohoff RM, Cardona AE. The myeloid cells of the central nervous system parenchyma. Nature 2010;468:253-62.

5. Anrather J, Iadecola C. Inflammation and Stroke: An Overview. Neurotherapeutics 2016;13:661-70.

6. Rodrigo R, Fernandez-Gajardo R, Gutierrez R, et al. Oxidative stress and pathophysiology of ischemic stroke: novel therapeutic opportunities. CNS Neurol Disord Drug Targets 2013;12:698-714.

7. Radermacher KA, Wingler K, Kleikers P, et al. The 1027th target candidate in stroke: Will NADPH oxidase hold up? Exp Transl Stroke Med 2012;4:11.

8. Moskowitz MA, Lo EH, Iadecola C. The science of stroke: mechanisms in search of treatments. Neuron 2010;67:181-98.

9. Fan LM, Cahill-Smith S, Geng L, et al. Aging-associated metabolic disorder induces Nox2 activation and oxidative damage of endothelial function. Free Radic Biol Med 2017;108:940-51.

10. Meng X, Wang M, Wang X, et al. Suppression of NADPH oxidase- and mitochondrion-derived superoxide by Notoginsenoside R1 protects against cerebral ischemiareperfusion injury through estrogen receptor-dependent activation of Akt/Nrf2 pathways. Free Radic Res 2014;48:823-38. 
11. Serrano F, Kolluri NS, Wientjes FB, et al. NADPH oxidase immunoreactivity in the mouse brain. Brain Res 2003;988:193-8.

12. Vallet $\mathrm{P}$, Charnay $\mathrm{Y}$, Steger K, et al. Neuronal expression of the NADPH oxidase NOX4, and its regulation in mouse experimental brain ischemia. Neuroscience 2005;132:233-8.

13. Zhang QG, Raz L, Wang R, et al. Estrogen attenuates ischemic oxidative damage via an estrogen receptor alphamediated inhibition of NADPH oxidase activation. J Neurosci 2009;29:13823-36.

14. Chen H, Song YS, Chan PH. Inhibition of NADPH oxidase is neuroprotective after ischemia-reperfusion. $\mathrm{J}$ Cereb Blood Flow Metab 2009;29:1262-72.

15. Warner TD, Nylander S, Whatling C. Anti-platelet therapy: cyclo-oxygenase inhibition and the use of aspirin with particular regard to dual anti-platelet therapy. Br J Clin Pharmacol 2011;72:619-33.

16. Taubert D, Kastrati A, Harlfinger S, et al. Pharmacokinetics of clopidogrel after administration of a high loading dose. Thromb Haemost 2004;92:311-6.

17. Kernan WN, Ovbiagele B, Black HR, et al. Guidelines for the prevention of stroke in patients with stroke and transient ischemic attack: a guideline for healthcare professionals from the American Heart Association/ American Stroke Association. Stroke 2014;45:2160-236.

18. Bhatt DL, Fox KA, Hacke W, et al. Clopidogrel and aspirin versus aspirin alone for the prevention of atherothrombotic events. N Engl J Med 2006;354:1706-17.

19. Arya V, Mahajan P, Saraf A, et al. Association of CYP2C19, CYP3A5 and GPIIb/IIIa gene polymorphisms with Aspirin and Clopidogrel Resistance in a cohort of Indian patients with Coronary Artery Disease. Int J Lab Hematol 2015;37:809-18.

20. Degrauwe S, Iglesias JF. Dual antiplatelet therapy for treatment and secondary prevention of coronary artery disease: indications, modalities and duration. Rev Med Suisse 2016;12:1022-6, 1028-34.

21. Lei XL, Chiou GC. Cardiovascular pharmacology of Panax notoginseng (Burk) F.H. Chen and Salvia miltiorrhiza. Am J Chin Med 1986;14:145-52.

22. Su P, Wang L, Du SJ, et al. Advance in studies of Panax notoginseng saponins on pharmacological mechanism of nervous system disease. Zhongguo Zhong Yao Za Zhi 2014;39:4516-21.

23. Zhao NN, Tang SM, Li XY, et al. Protective effects of combination of Xuesaitong and aspirin on cerebral ischemia and reperfusion injury in rats. Chinese Herbal Medicines 2018;10:223-30.
24. Ning N, Dang X, Bai C, et al. Panax notoginsenoside produces neuroprotective effects in rat model of acute spinal cord ischemia-reperfusion injury. J Ethnopharmacol 2012;139:504-12.

25. Zeng XS, Zhou XS, Luo FC, et al. Comparative analysis of the neuroprotective effects of ginsenosides $\mathrm{Rg} 1$ and Rb1 extracted from Panax notoginseng against cerebral ischemia. Can J Physiol Pharmacol 2014;92:102-8.

26. Zhu T, Wang L, Tian F, et al. Anti-ischemia/reperfusion injury effects of notoginsenoside R1 on small molecule metabolism in rat brain after ischemic stroke as visualized by MALDI-MS imaging. Biomed Pharmacother 2020;129:110470.

27. Jiang B, Li L, Chen Q, et al. Role of Glibenclamide in Brain Injury After Intracerebral Hemorrhage. Transl Stroke Res 2017;8:183-93.

28. Wang FJ, Wang SX, Chai LJ, et al. Xueshuantong injection (lyophilized) combined with salvianolate lyophilized injection protects against focal cerebral ischemia/reperfusion injury in rats through attenuation of oxidative stress. Acta Pharmacol Sin 2018;39:998-1011.

29. Shin HK, Lee SW, Choi BT. Modulation of neurogenesis via neurotrophic factors in acupuncture treatments for neurological diseases. Biochem Pharmacol 2017;141:132-42.

30. Smith-Dijak AI, Sepers MD, Raymond LA. Alterations in synaptic function and plasticity in Huntington disease. J Neurochem 2019;150:346-65.

31. Kim JT, Park MS, Choi KH, et al. Comparative Effectiveness of Aspirin and Clopidogrel Versus Aspirin in Acute Minor Stroke or Transient Ischemic Attack. Stroke. 2018;STROKEAHA118022691. [Epub ahead of print].

32. $\mathrm{He} \mathrm{W}, \mathrm{Xu} \mathrm{XJ}$. Attenuation of brain inflammatory response after focal cerebral ischemia/reperfusion with Xuesaitong injection in rats. Chin J Integr Med 2006;12:203-6.

33. Jiang $M$, Zhao $X$, Wang $L$, et al. Integrating candidate metabolites and biochemical factors to elucidate the action mechanism of Xue-sai-tong injection based on (1)H NMR metabolomics. J Chromatogr B Analyt Technol Biomed Life Sci 2016;1026:87-96.

34. Yao Y, Chen L, Xiao J, et al. Chrysin protects against focal cerebral ischemia/reperfusion injury in mice through attenuation of oxidative stress and inflammation. Int J Mol Sci 2014;15:20913-26.

35. Tang BL. Protein trafficking mechanisms associated with neurite outgrowth and polarized sorting in neurons. J Neurochem 2001;79:923-30.

36. Pang J, Peng J, Matei N, et al. Apolipoprotein E Exerts 
a Whole-Brain Protective Property by Promoting M1? Microglia Quiescence After Experimental Subarachnoid Hemorrhage in Mice. Transl Stroke Res 2018;9:654-68.

37. Bahar E, Kim JY, Yoon H. Quercetin Attenuates Manganese-Induced Neuroinflammation by Alleviating Oxidative Stress through Regulation of Apoptosis, iNOS/NF- $\kappa$ B and HO-1/Nrf2 Pathways. Int J Mol Sci 2017;18:1989.

38. Ito F, Yamada Y, Shigemitsu A, et al. Role of Oxidative Stress in Epigenetic Modification in Endometriosis. Reprod Sci 2017;24:1493-502.

39. Justicia C, Salas-Perdomo A, Perez-de-Puig I, et al. Uric Acid Is Protective After Cerebral Ischemia/Reperfusion in Hyperglycemic Mice. Transl Stroke Res 2017;8:294-305.

40. Olmez I, Ozyurt H. Reactive oxygen species and ischemic cerebrovascular disease. Neurochem Int 2012;60:208-12.

41. Uttara B, Singh AV, Zamboni P, et al. Oxidative stress and neurodegenerative diseases: a review of upstream and downstream antioxidant therapeutic options. Curr Neuropharmacol 2009;7:65-74.

42. Wingler K, Hermans JJ, Schiffers P, et al. NOX1, 2, 4, 5: counting out oxidative stress. Br J Pharmacol 2011;164:866-83.

43. Byrne JA, Grieve DJ, Bendall JK, et al. Contrasting roles of NADPH oxidase isoforms in pressure-overload versus angiotensin II-induced cardiac hypertrophy. Circ Res 2003;93:802-5.

44. Xiao L, Pimentel DR, Wang J, et al. Role of reactive oxygen species and $\mathrm{NAD}(\mathrm{P}) \mathrm{H}$ oxidase in alpha(1)adrenoceptor signaling in adult rat cardiac myocytes. Am J Physiol Cell Physiol 2002;282:C926-34.

45. Lin Y'T, Wu YC, Sun GC, et al. Effect of Resveratrol on Reactive Oxygen Species-Induced Cognitive Impairment in Rats with Angiotensin II-Induced Early Alzheimer's Disease †. J Clin Med 2018;7:329.

46. Li JM, Fan LM, Christie MR, et al. Acute tumor necrosis factor alpha signaling via NADPH oxidase in microvascular endothelial cells: role of $\mathrm{p} 47$ phox phosphorylation and binding to TRAF4. Mol Cell Biol 2005;25:2320-30.

47. Brewster UC, Setaro JF, Perazella MA. The reninangiotensin-aldosterone system: cardiorenal effects and implications for renal and cardiovascular disease states. Am J Med Sci 2003;326:15-24.

48. Kusaka I, Kusaka G, Zhou C, et al. Role of AT1 receptors and $\mathrm{NAD}(\mathrm{P}) \mathrm{H}$ oxidase in diabetes-aggravated ischemic brain injury. Am J Physiol Heart Circ Physiol 2004;286:H2442-51.
49. Cos P, De Bruyne T, Apers S, et al. Phytoestrogens: recent developments. Planta Med 2003;69:589-99.

50. Zhang L, Li Z, Feng D, et al. Involvement of Nox2 and Nox4 NADPH oxidases in early brain injury after subarachnoid hemorrhage. Free Radic Res 2017;51:316-28.

51. Zhao SC, Ma LS, Chu ZH, et al. Regulation of microglial activation in stroke. Acta Pharmacol Sin 2017;38:445-58.

52. Mayo L, Cunha AP, Madi A, et al. IL-10-dependent Tr1 cells attenuate astrocyte activation and ameliorate chronic central nervous system inflammation. Brain 2016;139:1939-57.

53. Wu BN, Wu J, Hao DL, et al. High serum sICAM-1 is correlated with cerebral microbleeds and hemorrhagic transformation in ischemic stroke patients. Br J Neurosurg 2018;32:631-6.

54. Wang G, Zhang J, Hu X, et al. Microglia/macrophage polarization dynamics in white matter after traumatic brain injury. J Cereb Blood Flow Metab 2013;33:1864-74.

55. Corzo CA, Cotter MJ, Cheng P, et al. Mechanism regulating reactive oxygen species in tumor-induced myeloid-derived suppressor cells. J Immunol 2009;182:5693-701.

56. Murray PJ. The JAK-STAT signaling pathway: input and output integration. J Immunol 2007;178:2623-9.

57. Hung CC, Lin CH, Chang H, et al. Astrocytic GAP43 Induced by the TLR4/NF-kappaB/STAT3 Axis Attenuates Astrogliosis-Mediated Microglial Activation and Neurotoxicity. J Neurosci 2016;36:2027-43.

58. Ben Haim L, Ceyzeriat K, Carrillo-de Sauvage MA, et al. The JAK/STAT3 pathway is a common inducer of astrocyte reactivity in Alzheimer's and Huntington's diseases. J Neurosci 2015;35:2817-29.

59. Mudter J and Neurath MF. Il-6 signaling in inflammatory bowel disease: pathophysiological role and clinical relevance. Inflamm Bowel Dis 2007;13:1016-23.

Cite this article as: Zhu T, Meng XB, Dong DX, Zhao LY, Qu MW, Sun GB, Sun XB. Xuesaitong injection (lyophilized) combined with aspirin and clopidogrel protect against focal cerebral ischemic/reperfusion injury in rats by suppressing oxidative stress and inflammation and regulating the NOX2/ IL-6/STAT3 pathway. Ann Palliat Med 2021;10(2):1650-1667. doi: 10.21037/apm-20-1681 
A

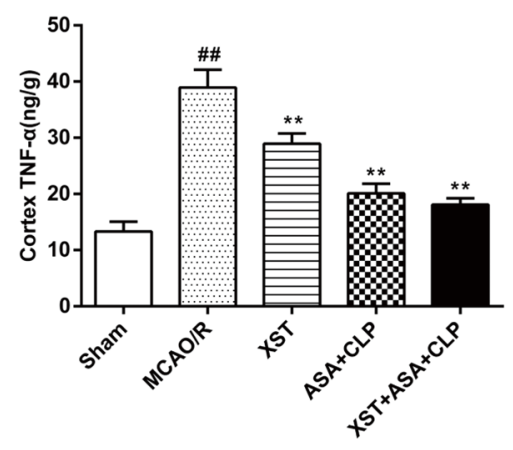

D

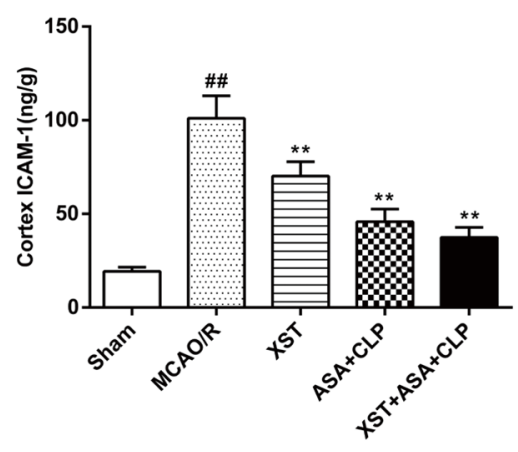

G

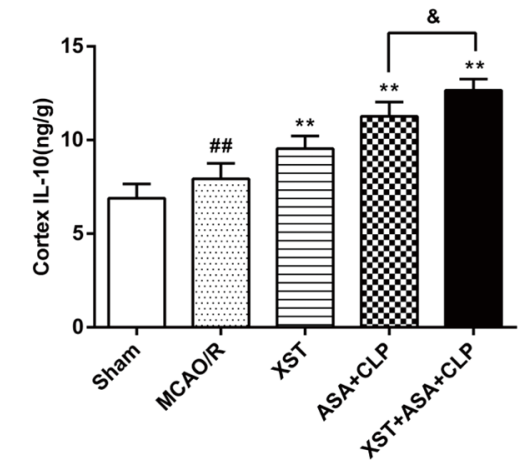

B

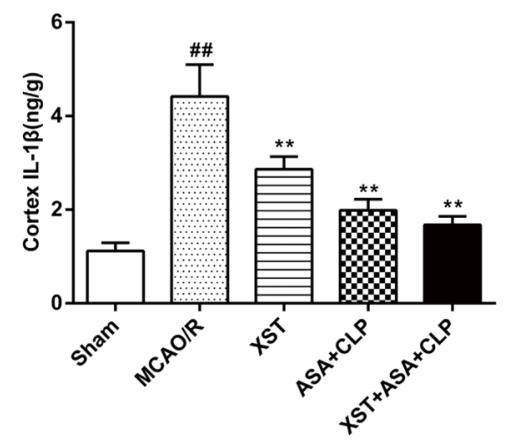

E

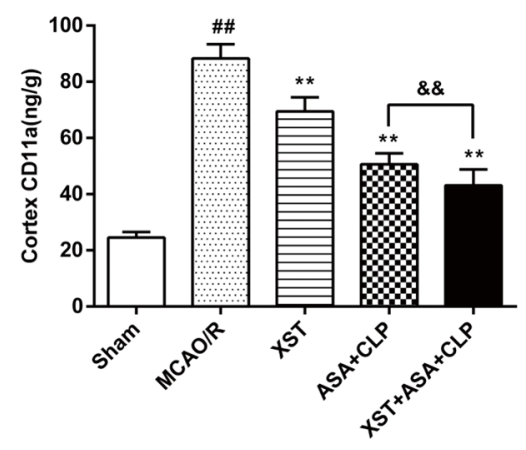

H

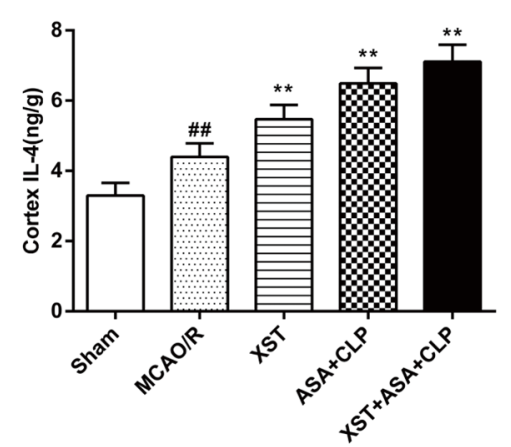

C

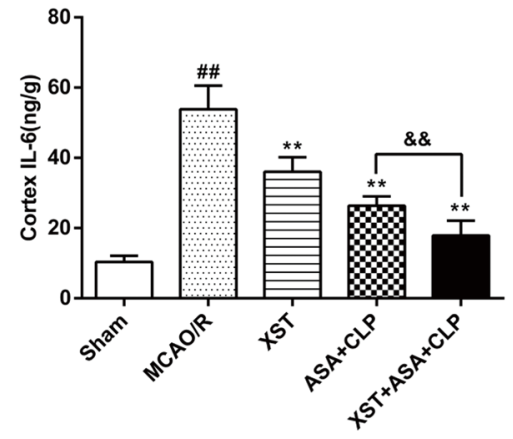

F

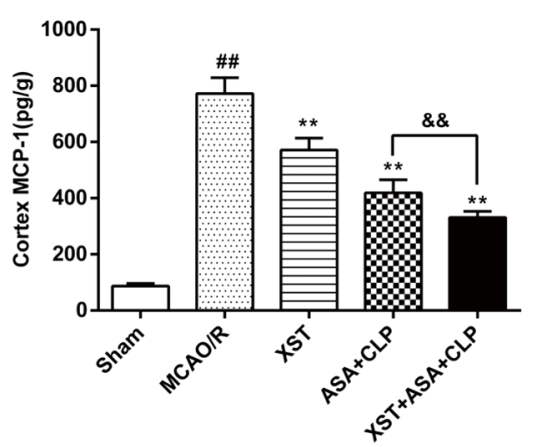

Figure S1 XST+ASA+CLP inhibits inflammatory response in rat cortex with MCAO/R. (A) Effect of XST+ASA+CLP treatment on cortex TNF- $\alpha$ (A), IL-1 $\beta$ (B), IL-6 (C), ICAM-1 (D), CD11a (E), MCP-1 (F), IL-10 (G) and IL-4 (H) in rats with MCAO/R. Values are represented as means $\pm \mathrm{SD}$ for 6 mice in each group. ${ }^{* *}, \mathrm{P}<0.01$, compared with the $\mathrm{MCAO} / \mathrm{R}$ group; ${ }^{\prime \prime \prime}, \mathrm{P}<0.01$, compared with the sham group; ${ }^{\&}, \mathrm{P}<0.05 ;{ }^{\& \&}, \mathrm{P}<0.01$, compared with the ASA+CLP group. XST, Xuesaitong injection (lyophilized); ASA, aspirin; CLP, clopidogrel; $\mathrm{MCAO} / \mathrm{R}$, middle cerebral artery occlusion/reperfusion; TNF- $\alpha$, tumor necrosis factor-alpha; IL-1 $\beta$, interleukin-1 beta; IL-6, interleukin-6; ICAM-1, intercellular adhesion molecule-1; MCP-1, monocyte chemoattractant protein-1; IL-10, interleukin-10; IL-4, interleukin-4. 
A

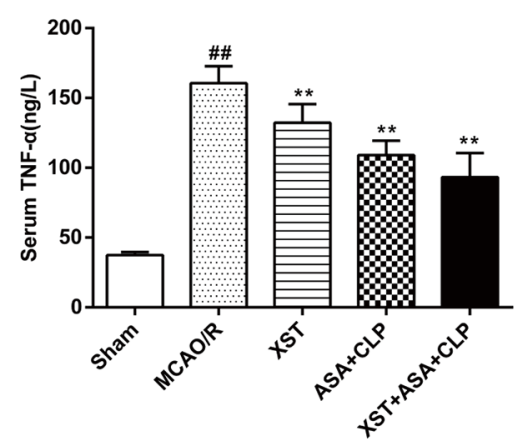

D

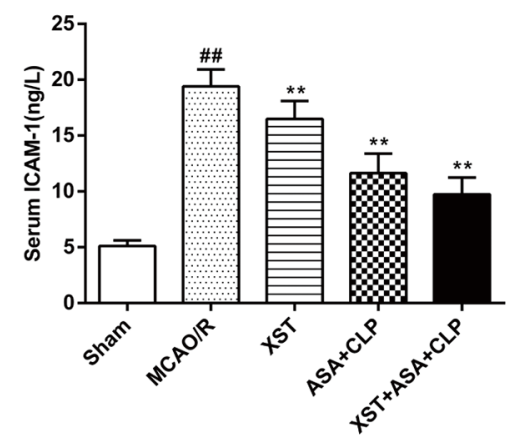

G

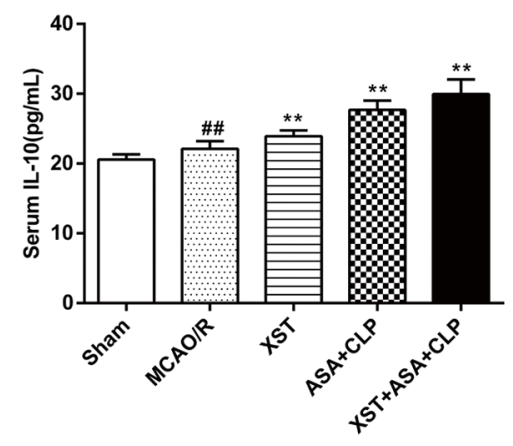

B

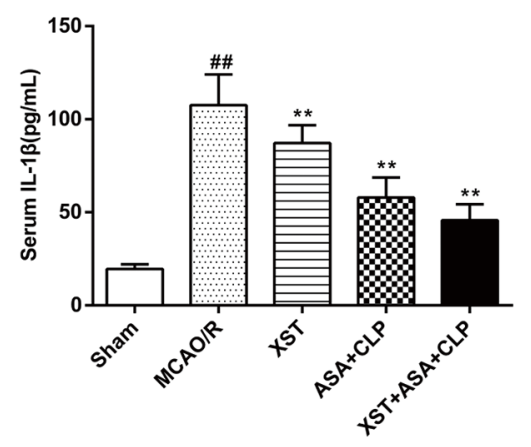

E

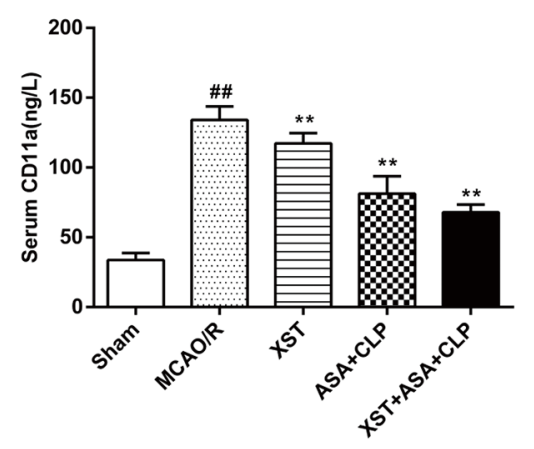

H

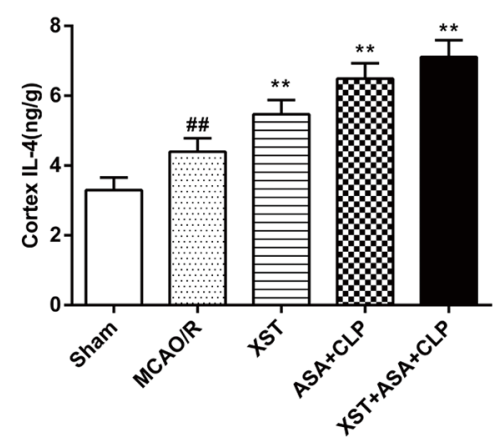

C

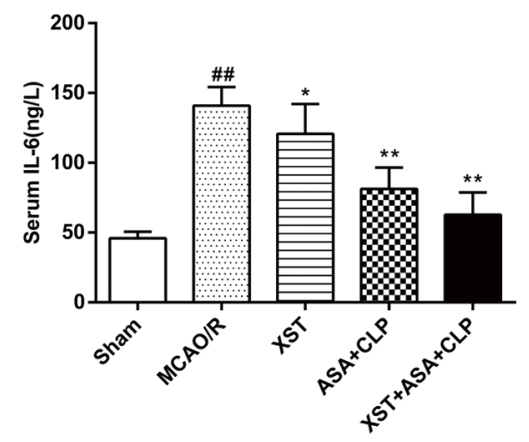

F

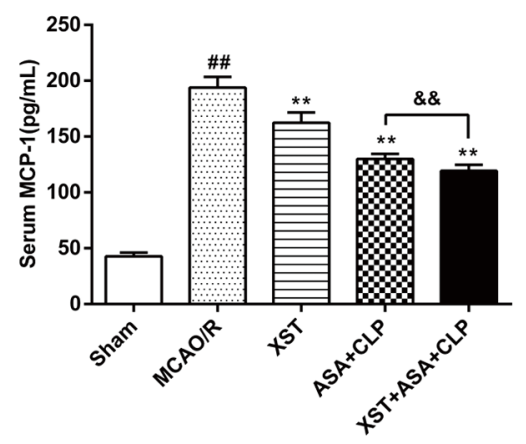

Figure S2 XST+ASA+CLP inhibits inflammatory response in rat serum with MCAO/R. (A) Effect of XST+ASA+CLP treatment on serum TNF- $\alpha$ (A), IL-1 $\beta$ (B), IL-6 (C), ICAM-1 (D), CD11a (E), MCP-1 (F), IL-10 (G) and IL-4 (H) in rats with MCAO/R. Values are represented as means $\pm \mathrm{SD}$ for 6 mice in each group. ${ }^{*}, \mathrm{P}<0.05 ;{ }^{* *}, \mathrm{P}<0.01$, compared with the MCAO/R group; ${ }^{\# \#}, \mathrm{P}<0.01$, compared with the sham group; ${ }^{\& \&}, \mathrm{P}<0.01$, compared with the ASA+CLP group. XST, Xuesaitong injection (lyophilized); ASA, aspirin; CLP, clopidogrel; MCAO/R, middle cerebral artery occlusion/reperfusion; TNF- $\alpha$, tumor necrosis factor-alpha; IL-1 $\beta$, interleukin-1 beta; IL-6, interleukin-6; ICAM-1, intercellular adhesion molecule-1; MCP-1, monocyte chemoattractant protein-1; IL-10, interleukin-10; IL-4, interleukin-4. 
A

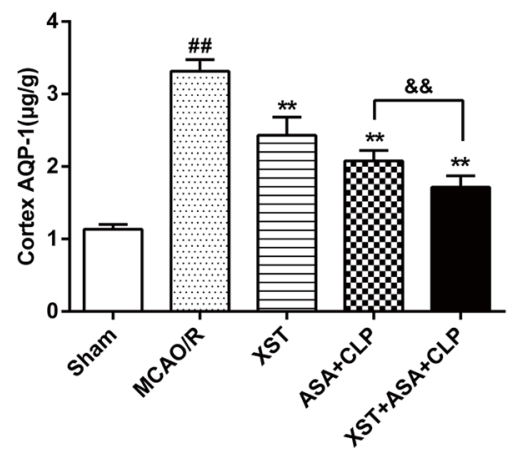

D

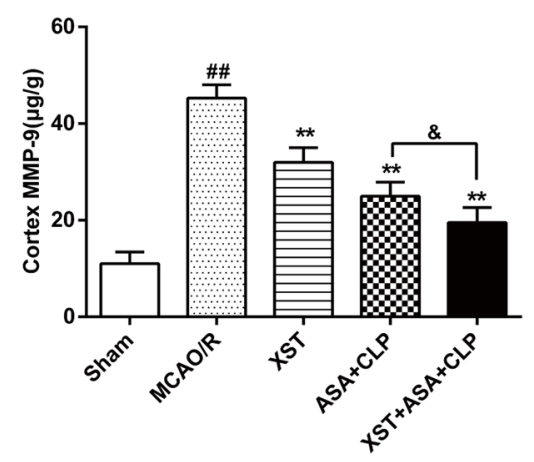

B

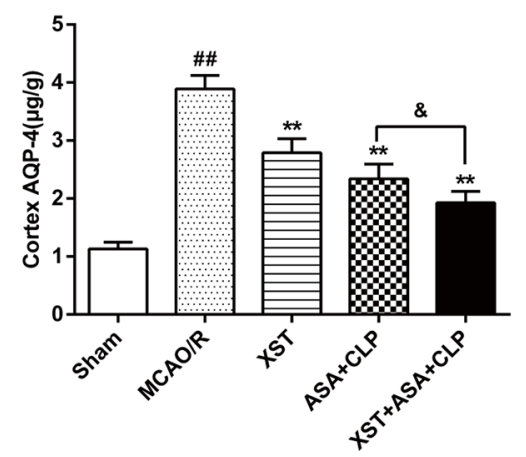

E

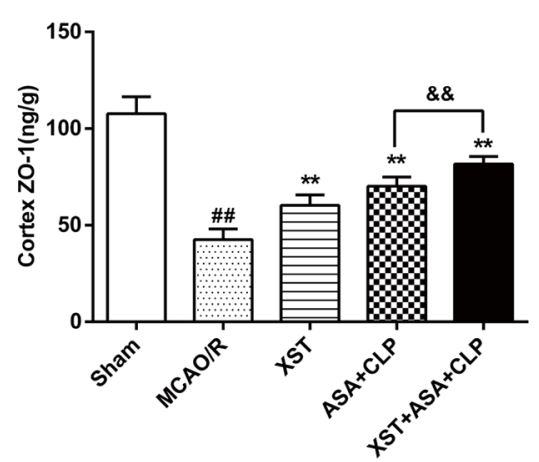

C

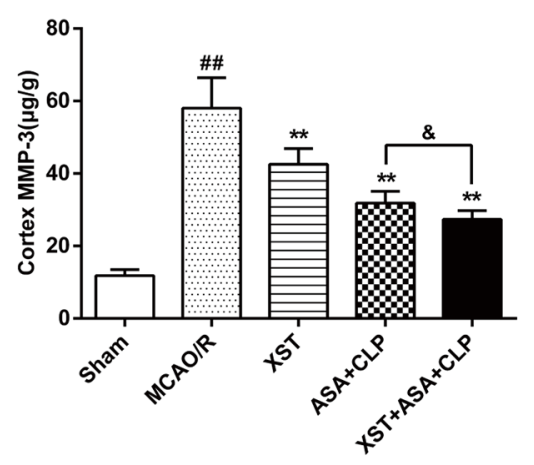

Figure S3 XST+ASA+CLP improves blood-brain barrier permeability in rat cortex with MCAO/R. (A) Effect of XST+ASA+CLP treatment on cortex AQP-1 (A), AQP-4 (B), MMP-3 (C), MMP-9 (D) and ZO-1 (E) in rats with MCAO/R. n=6 in sham group; n=8 in other groups. **, $\mathrm{P}<0.01$, compared with the MCAO/R group; ${ }^{\#}, \mathrm{P}<0.01$, compared with the sham group; ${ }^{\&}, \mathrm{P}<0.05,{ }^{\& \&}, \mathrm{P}<0.01$, compared with the ASA+CLP group. XST, Xuesaitong injection (lyophilized); ASA, aspirin; CLP, clopidogrel; MCAO/R, middle cerebral artery occlusion/ reperfusion; AQP-1, aquaporin-1; AQP-4, aquaporin-4; MMP-3, matrix metalloproteinase 3; MMP-9, matrix metalloproteinase 9; ZO-1, zona occludens 1. 